\title{
A sunflower WRKY transcription factor stimulates the mobilization of seed- stored reserves during germination and post-germination growth
}

Jesica Raineri 1

Matías D. Hartman 1

Raquel L. Chan 1

Alberto A. Iglesias 1

Karina F. Ribichich 1,*

Phone +54-342-4511370

Email kribi@fbcb.unl.edu.ar

1 Instituto de Agrobiotecnología del Litoral, UNL, CONICET; FBCB, UNL; Centro Científico Tecnológico CONICET Santa Fe, Colectora Ruta Nacional $\mathrm{N}^{\circ} 168 \mathrm{~km}$. 0, Paraje El Pozo (3000), Santa Fe, Argentina

\section{Abstract}

\section{Key message}

The sunflower transcription factor HaWRKY10 stimulates reserves mobilization in Arabidopsis. Gene expression and enzymes activity assays indicated that lipolysis and gluconeogenesis were increased. Microarray results suggested a parallelism in sunflower.

\section{Abstract}

Germinating oilseeds converts stored lipids into sugars, and thereafter in metabolic energy that is used in seedling growth and establishment. During 
germination, the induced lipolysis linked to the glyoxylate pathway and gluconeogenesis produces sucrose, which is then transported to the embryo and driven through catabolic routes. Herein, we report that the sunflower transcription factor HaWRKY10 regulates carbon partitioning by reducing carbohydrate catabolism and increasing lipolysis and gluconeogenesis. HaWRKY10 was regulated by abscisic acid and gibberellins in the embryo leaves $48 \mathrm{~h}$ after seed imbibition and highly expressed during sunflower seed germination and seedling growth, concomitantly with lipid mobilization. Sunflower leaf disks overexpressing HaWRKY10 showed repressed expression of genes related to sucrose cleavage and glycolysis compared with controls. Moreover, HaWRKY10 constitutive expression in Arabidopsis seeds produced higher decrease in lipid reserves, whereas starch and sucrose were more preserved compared with wild type. Gene transcripts abundance and enzyme activities involved in stored lipid mobilization and gluconeogenesis increased more in transgenic than in wild type seeds $36 \mathrm{~h}$ after imbibition, whereas the negative regulator of lipid mobilization, $A B I 4$, was repressed. Altogether, the results point out a functional parallelism between tissues and plant species, and reveal HaWRKY10 as a positive regulator of storage reserve mobilization in sunflower.

AQ1

$\mathrm{AQ} 2$

\section{Keywords}

Sunflower

Reserves mobilization

Germination

Arabidopsis

Transcription factor

WRKY

Communicated by R. J. Rose.

Electronic supplementary material

The online version of this article (doi:10.1007/s00299-016-2002-2) contains 
supplementary material, which is available to authorized users.

\section{Introduction}

Seeds store carbon sources (carbohydrates, oils, and proteins) that are consumed during germination and post-germination growth (Bewley and Black 1994). Oil crops store mainly oil as triacylglycerols (TAGs) (Thelen and Ohlrogge 2002), in either the endosperm or the cotyledons depending on whether the endosperm persists or not in the mature seed (Nonogaki 2008). Some seeds, like those of Arabidopsis thaliana (L.) Heynh. (Arabidopsis) or Helianthus annuus L. (sunflower), store carbohydrates as a roughly minor component (Eastmond and Graham 2001; Meyer et al. 2012), which are quickly consumed during the first 2 days after imbibition, whereas TAGs levels decrease gradually (Balasaraswathi and Sadasivam 1997; Pritchard et al. 2002). Lipids are consumed through their conversion to carbohydrates. During germination, lipolysis is followed by the glyoxylate and gluconeogenesis pathways mainly conducting to the production of glucose and then sucrose, which are used for seedling growth. This particular pipeline occurs both in endospermic (e.g., castor bean, oil palm, and Arabidopsis) and in non-endospermic (e.g., canola, soybean, and sunflower) oil plants (Eckardt 2005; Yan et al. 2014).

The available information about the regulation of reserves mobilization in dicotyledons (including oilseeds) is limited. In general, lipases are only detected after imbibition, but for several of them, the relevance in TAGs breakdown has not been clearly established (Bewley et al. 2013). In Arabidopsis, germination is supported by the limited stored sugars, whereas lipolysis impairment has very little effect on this event. However, subsequent growth and survival are dramatically dependent on oil breakdown (Cornah et al. 2004; Kelly et al. 2011) and gluconeogenesis (Rylott et al. 2003). The main seed lipase sugar-dependent1 (SDP1), together with SDP1-like (SDP1L), is responsible for around $95 \%$ of TAGs hydrolysis in Arabidopsis seeds (Eastmond 2006; Kelly et al. 2011). In the double mutant sdp1-5 $s d p 1 L-2$, growth was severely affected and the percentage of seedling establishment dropped down to less than $20 \%$ (Kelly et al. 2011). Seedling establishment was also directly correlated with the phosphoenolpyruvate 
carboxykinase (PEPCK) activity, one of the key enzymes in gluconeogenesis (Rylott et al. 2003).

Abscisic acid (ABA) and gibberellins (GAs) regulate in an opposite way the timing of the germination onset (Corbineau et al. 2014; Finch-Savage and Leubner-Metzger 2006), which includes, at least in part, carbon reserves mobilization (Penfield et al. 2004; Pritchard et al. 2002; Rylott et al. 2001; Yan et al. 2014). The earliest and more explored model of carbon usage was built with studies in monocotyledons and is represented by cereal seeds, mainly from barley (Gubler et al. 1995, 2002). The seed cotyledon of barley synthesizes and secretes GAs to the aleurone layer of the endosperm. There, GAs induce the synthesis of $\alpha$-amylase through the transcriptional activator GAMYB, which is secreted to the amylaceous endosperm. The embryo employs the carbohydrates released from starch degradation for its growth. Conversely, ABA inhibits the pathway by repressing the signal translation and, probably, GAs synthesis (Gómez-Cadenas et al. 2001; Gubler et al. 2005 ). Probably, these processes occur in a similar way in dicotyledons. In Arabidopsis, the aleurone layer responds to GAs, and receptors and effectors are conserved with cereals (Gocal et al. 2001; Linkies and Leubner-Metzger 2012). Moreover, ABA and GAs have opposite roles in storage oil breakdown in the embryo (Penfield et al. 2004). ABA inhibits it by inducing the expression of the AP2-type transcription factor (TF) ABSCISIC ACIDINSENSITIVE4 (ABI4), whereas GAs are required for the lipid breakdown (Penfield et al. 2006). This control appears to be exerted at the transcriptional and the post-transcriptional levels, considering that the stimulation of lipid breakdown is produced with a coordinated increase in genes expression and enzyme activities directly involved in the partitioning of the reserves (Rylott et al. 2001).

Members of plant WRKY TFs family have been involved in plant responses to biotic and abiotic stresses (Chen et al. 2010; Eulgem and Somssich 2007; Rushton et al. 2010; Tripathi et al. 2014), the regulation of growth and development processes (Ding et al. 2014; Rushton et al. 2010), and the biosynthesis of secondary metabolites in plants (Schluttenhofer and Yuan 2015 ). Germination and post-germination growth are among the processes in which these TFs are key regulators, which include carbohydrates seed maintenance and anabolism, at least in monocotyledons (Mena et al. 2002; 
Rushton et al. 1995; Sun et al. 2003; Xie et al. 2006; Zhang et al. 2004). The role of WRKY TFs in seed reserves mobilization in dicotyledons is less evident. AtWRKY18, AtWRKY40, AtWRKY41, AtWRKY60, AtWRKY2, and AtWRKY63 act as regulators of seed germination via ABA-dependent or independent pathways; however, these genes have not been associated with reserves mobilization (Ding et al. 2014; Rushton et al. 2010). AtWRKY27 was detected as induced by the DELLA-protein RGA in microarray and chromatin immunoprecipitation assays, and could repress the induction of the $\alpha$-amylase in Arabidopsis (Zentella et al. 2007). So far, AtWRKY40 is the only WRKY TF that has been related to lipid catabolism. In the atwrky40-1 mutant, the expression of the gene encoding the enzyme that catalyzes the $\beta$ oxidation of fatty acids (KAT2) is induced; however, its stimulation by ABA was abolished. This observation suggested that KAT2 might function downstream of AtWRKY40, linking this TF with lipid catabolism and ABA signaling (Jiang et al. 2011).

HaWRKY10 is a sunflower member of the WRKY family resolved in group IId (Giacomelli et al. 2010). To functionally characterize this TF, expression patterns of the encoding gene in standard growth conditions and in plants treated with different hormones were determined. The observations performed in cotyledons (embryo leaves and first leaves of the seedling) led us to further investigate its role in germination. To investigate gene regulatory networks that could be modulated by HaWRKY10, microarray experiments were conducted using sunflower leaf disks transient transformation (Manavella and Chan 2009; Manavella et al. 2008). In parallel, transgenic plants of Arabidopsis (another oilseed) expressing HaWRKY10 under the control of the 35S promoter were obtained. The results of the analysis performed with transient and stable transformations, followed by molecular and phenotypical analyses, support HaWRKY10 as a positive regulator of lipolysis and gluconeogenesis during germination and post-germination processes, probably via the repression of $A B I 4$. To the best of our knowledge, this is the first report connecting a WRKY transcription factor with lipid catabolism and gluconeogenesis.

\section{Materials and methods}

\section{Cloning of the HaWRKY1O cDNA}


HaWRKY10 cDNA was cloned from sunflower leaves RNA as template in three steps. First, the $5^{\prime}$-end was amplified by reverse transcription followed by PCR using W10RACE-F and W10DEG-R oligonucleotides designed over the uncomplete EST sequences in Heliagene database (https://www.heliagene.org). Then, a random amplification of the $3^{\prime}$ cDNA end (3'RACE) (Frohman et al. 1988) was conducted using PolyT-V oligonucleotide $\left(0.5 \mathrm{~min}\right.$ at $94{ }^{\circ} \mathrm{C}$, and 5 cycles of $0.5 \mathrm{~min}$ at $94{ }^{\circ} \mathrm{C}, 0.5 \mathrm{~min}$ at $55^{\circ} \mathrm{C}, 3 \mathrm{~min}$ at $72{ }^{\circ} \mathrm{C}$ ), and W10RACE-F oligonucleotide ( 25 cycles of $0.5 \mathrm{~min}$ at $94{ }^{\circ} \mathrm{C}, 0.5 \mathrm{~min}$ at $55^{\circ} \mathrm{C}$, and $2 \mathrm{~min}$ at $72{ }^{\circ} \mathrm{C}$ plus 1 final cycle of 10 min at $72{ }^{\circ} \mathrm{C}$ ). Finally, the complete cDNA was cloned by PCR using HaW10ADNc-F and HaW10ADNc-R (Supplementary Table S1). The 5'-end and the complete cDNA were amplified following the same program (2 min at $94{ }^{\circ} \mathrm{C}$, and 35 cycles of $0.5 \mathrm{~min}$ at $94{ }^{\circ} \mathrm{C}, 0.5 \mathrm{~min}$ at $55^{\circ} \mathrm{C}$ and $2 \mathrm{~min}$ at $72^{\circ} \mathrm{C}$ ). Since the protein coding sequence amplified in this study and used in the assays is coincident with a sequence from the Heliagene database, the same accession number for the HaWRKY10 cDNA was maintained (accession number: HaT131041747; https://www.heliagene.org/HaT131/). The cDNA was cloned into a pGEM T-Easy vector (Promega) and then subcloned in pBI122 (Capella et al. 2014) under the control of the 35S CaMV promoter.

\section{Plant material and growth conditions}

For microarray experiments, Helianthus annuus L. (sunflower CF31, Advanta) seeds were grown on soil in $8-\mathrm{cm}$ diameter $\times 7-\mathrm{cm}$ height pots, one plant per pot. The plants were irrigated with $0.5 \times$ Hoagland solution. For gene expression analyses and carbon sources extraction, sunflower seeds pretreated with fungicide were sown on moistened paper towels. To collect sunflower seeds phenologically synchronized, time course imbibition curves were previously registered, and a homogenous population of seeds (the same water content increase rate and time of germination) was selected and used in the following experiments.

Arabidopsis thaliana Heyhn. ecotype Columbia (Col-0) and the transgenic seeds transformed with the $35 \mathrm{~S}$ :HaWRKY10 construct were subjected to cold stratification during 2 days, and then sown on a mesh laid on $0.5 \times$ Murashige and Skoog medium with $0.8 \%$ agar in Petri dishes. 
Seeds and plants were grown in a chamber at $22-24{ }^{\circ} \mathrm{C}$, under an irradiance of $120-150 \mu \mathrm{E} \mathrm{m}^{-2} \mathrm{~s}^{-1}$, with an LD 16:8-h photoperiod. In all the experiments, seeds harvested at the same time, air drying in the darkness and stored at $4{ }^{\circ} \mathrm{C}$, were used. At the periods indicated in the figures, samples were collected and frozen with liquid nitrogen until evaluation.

\section{Transient transformation of sunflower leaf disks}

Leaf disks from V3 (three pairs of leaves) sunflower plants were used as samples. Transient transformation was done as previously reported (Manavella and Chan 2009). Agrobacterium tumefaciens strain LBA4404 (Hoekema et al. 1983) previously transformed with empty pBI122 (Capella et al. 2014) or with HaWRKY10 in pBI122, together with strain GV3101 with the construct pBIN61:p19 (Voinnet et al. 2003), expressing a gene for posttranscriptional gene silencing from Tomato bushy stunt virus, was used in cotransformation experiments. A mix of $0.5 A_{600}$ strain LBA4404 culture with $1 / 10$ of $0.5 A_{600}$ strain GV3101 was used for vacuum infiltration. Forty eight plants pooled in six biological replicates were used for total RNA extractions.

\section{RNA isolation, reverse transcription, and quantitative PCR}

Total RNA was isolated following the Trizol ${ }^{\circledR}$ reagent protocol (Invitrogen TM). Absorbance ratios $\left(A_{260} / 280, A_{260} / 230\right)$ were determined to check RNA purity, and agarose gel electrophoresis in denaturing conditions were performed to control RNA integrity. Genomic or plasmid DNA contamination was assessed by PCR using specific primers of ACTIN for genomic and HaWRKY10 for plasmid contaminations. Reverse transcription was conducted using $0.5-1 \mu \mathrm{g}$ total RNA and M-MLV RT (Promega ${ }^{\mathrm{TM}}$ ) according to manufacturer' instructions. Quantitative PCR, including PCR cycles, controls, and methods, was carried out as previously described by Raineri et al. (2015) using specific designed oligonucleotides (Supplementary Table S1).

\section{Microarray experiment, data analysis, and validation}

A sunflower oligonucleotide-based chip, with 40,169 probes plus controls 
designed and validated (Fernandez et al. 2012; Moschen et al. 2016), was used for Agilent three replicates one-color microarray assay. An experiment with partially balanced incomplete block design was used to compare samples with or without overexpression of HaWRKY10 cDNA (accession number: GSE80675; http:/www.ncbi.nlm.nih.gov/geo/). RNA samples were evaluated according to quality standards described in the RNA extraction protocol. Final integrity (RIN >9) and purity were checked with an RNA 6000 Nano Bioanalyzer (Agilent Technologies, Palo Alto, CA, USA) at the Prince Felipe Research Center (Valencia, Spain), and three samples per treatment with the highest quality were used in the assay.

Differential gene expression between control and sunflower leaf disks overexpressing HaWRKY10 ( $\mathrm{HaWRKY10} \mathrm{OX)} \mathrm{was} \mathrm{determined} \mathrm{using} \mathrm{limma}$ package (linear models for microarray data) and the empirical Bayes approach (Smyth 2004), a $P$ value $<0.001$ and a fold change threshold of \pm 1.5 , provided with Babelomics 4 platform (Al-Shahrour et al. 2005). Annotation was upgraded using the tools integrated in the Blast2GO package (Conesa et al. 2005). The node scores were computed as score $=\sum_{\text {GOs }} \operatorname{seq} x \propto^{\text {dist }}$, where seq is the number of different sequences annotated at a child GO term and dist is the distance to the node of the child. Description of Arabidopsis putative orthologues was retrieved by PANTHER (Mi et al. 2013). The SCAVENGER tool provided by the Mapman software (Usadel et al. 2009) was used to map Arabidopsis putative orthologues of differentially expressed sunflower genes according to their function. Data about seed genes expression were extracted from openly available databases for sunflower and Arabidopsis (https://www.heliagene.org/Ha412T41, http://bar.utoronto.ca/efp/cgi-bin/efpWeb.cgi), considering the parameters used in the respective repositories. Cutoffs were adjusted according to the values provided by the databases. For sunflower, a gene was considered as expressed in seeds if the reads per kilobase of transcript per million mapped reads (RPKM ) were above 1.5 (around 100 counts); whereas for Arabidopsis, the same consideration was made if its fold-change compared with the control was above 1.5. Differential expression results were challenged by reverse transcription followed by quantitative PCR (RT-qPCR) using specific oligonucleotides (Supplementary Table S1). 


\section{Arabidopsis transformation}

Agrobacterium tumefaciens strain LBA4404 transformed with the 35S:HaWRKY10 cloned in pBI122 was used to obtain transgenic Arabidopsis plants by the floral dip procedure (Clough and Bent 1998). Fifteen homozygous and independent plant lines were obtained, and three of them (T3 homozygous lines) with roughly high transgene expression levels were chosen to pursue the characterization. WT plants were used as controls.

\section{Lipids, fatty acids, and oil body's extraction and quantification}

The extraction and quantification of lipids by gravimetry was based on the technique described elsewhere (Siloto et al. 2006) and adapted to microtube volumes. Around $25 \mathrm{mg}$ of seeds were ground and incubated with $400 \mu \mathrm{l}$ of isopropanol, $10 \mathrm{~min}$, at $65^{\circ} \mathrm{C}$, and then letting them dry. The three extractions done following the protocol was separated by centrifugations at $6700 \mathrm{~g}$ during $5 \mathrm{~min}$. All the fractions were collected in a unique previously weighted microtube (W1), and after drying of the sample, it was weighted again (W2). Lipids were expressed as W2 - W1/mg tissue. The initial sample weight was increased up to five times in post-germination seeds.

Fatty acids were extracted from the samples as indicated above and analyzed by gaseous chromatography by a specialized service (Ref. No. 601329/AU, Food Science and Nutrition Department, Dr. Claudio Bernal, FBCB, UNL, Santa Fe, Argentina).

Oil bodies were separated by a flotation centrifugation method according to van Rooijen and Moloney (van Rooijen and Moloney 1995) with minor changes. Twenty milligrams of Arabidopsis seeds were grounded with $1 \mathrm{ml}$ of extraction solution (0.4-M sucrose, $0.5-\mathrm{M} \mathrm{NaCl}, 50-\mathrm{mM}$ Tris- $\mathrm{HCl} \mathrm{pH}$ 8.0) and centrifuged at $2300 \mathrm{~g}$ during $5 \mathrm{~min}$. Floating oil bodies were washed

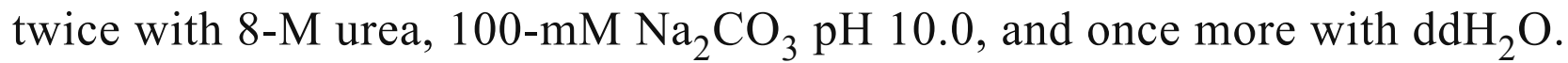
Oleosins were resolved by PAGE-SDS (Laemmli 1970).

\section{Carbon sources, protein, and chlorophyll extraction and quantification}

Glucose, sucrose, starch, and chlorophyll in plant extracts were quantified as 
previously described (Raineri et al. 2015). For Arabidopsis carbon sources, around $50 \mathrm{mg}$ of seeds were used by each sample. For sunflower, a pool of three seeds was used for each sample.

Proteins were extracted from $20 \mathrm{mg}$ of each sample, homogenized with liquid nitrogen in extraction buffer [50-mM sodium phosphate $\mathrm{pH} 7.0,10-\mathrm{mM}$ EDTA pH 8.0, 0.125 \% (w/v) SDS, $10 \mathrm{mM} \beta$-mercaptoethanol, $0.125 \%$ (v/v) Triton X-100, $1 \mathrm{mM}$ PMSF], centrifuged $3800 \mathrm{~g}$ during $15 \mathrm{~min}$ and quantified in the supernatant according to Bradford (1976) using BSA as standard.

\section{Enzyme activity assays}

Phosphoenolpyruvate carboxykinase (PEPCK, EC 4.1.1.32) and fructose-1,6bisphosphate phosphatase (FBPase, EC 3.1.3.11) activities were measured in protein extracts obtained from 50 to $100 \mathrm{mg}$ of 36 h.a.i. Arabidopsis seeds homogenized in a precooled mortar, with 200-400 $\mu 1$ 200-mM Bicine-KOH (pH 9.0) and 25-mM DTT. The homogenates were centrifuged for $10 \mathrm{~min}$ at $14,000 \mathrm{~g}$, and supernatants were collected for enzymatic determinations. One unit of activity is the amount of enzyme that catalyzes the formation of $1 \mu \mathrm{mol}$ product per min at $25^{\circ} \mathrm{C}$ under the specified assay conditions.

PEPCK activity, in the carboxylase direction, was measured by a continuous assay in which the oxaloacetic acid produced by PEPCK was immediately reduced to malate, through the action of coupled malate dehydrogenase (Malone et al. 2007). The oxidation of NADH by malate dehydrogenase was measured at $340 \mathrm{~nm}$ using a spectrophotometer. The reaction medium contained 100-mM HEPES pH 7.5, 5-mM 2-ME, 20-mM PEP, 0.3-mM ADP, 4-mM $\mathrm{MgCl}_{2}, 1-\mathrm{mM} \mathrm{MnCl}_{2}, 100-\mathrm{mM} \mathrm{KHCO}_{3}, 0.25-\mathrm{mM} \mathrm{NADH}$, and $0.5 \mathrm{U}$ malate dehydrogenase, and an appropriate amount of sample.

FBPase activity was measured using the method developed for ADP-glucose pyrophosphorylase (Fusari et al. 2006), where Pi dosage is performed by the formation of a phosphomolybdate-Malachite Green complex thereafter quantified at $650 \mathrm{~nm}$. The reaction medium contained 50-mM HEPES pH 7.5, 0.1-mM FBP, and 5-mM $\mathrm{MgCl}_{2}$ and an appropriate amount of sample.

Sucrose synthase (SUS, EC 2.4.1.13) cleaving activity was measured in 
proteins extracts obtained from $50 \mathrm{mg}$ of 36 h.a..i. Arabidopsis seeds resuspended at $4{ }^{\circ} \mathrm{C}$ in $100 \mu 1$ of $100-\mathrm{mM}$ Hepes (pH 7.5) and 5-mM DTT. The homogenate was subjected to centrifugation for $15 \mathrm{~min}$ at $12,000 \mathrm{~g}$ and $4{ }^{\circ} \mathrm{C}$, and supernatants were collected. SUS activity was measured in the breakdown direction (UDP-glucose synthesis) in a medium containing 50mM HEPES pH 7.0, 5-mM UDP, and 400-mM sucrose, and an appropriate amount of sample. After that, fructose was quantified by a microplate adapted-Somogy-Nelson method (Green et al. 1989).

\section{Hormone assays}

Arabidopsis seeds, previously subjected to cold stratification during 2 days, were sown on Petri dishes with $0.5 \times$ Murashige and Skoog medium in $0.8 \%$ agar with or without $\mathrm{ABA}, \mathrm{GA}_{3}$, or both hormones as indicated in the figures, and then transferred to the growth chamber. Gene expression and percentage of germinated seeds with expanded cotyledons were registered at the times indicated in the figures.

Seven-day-old sunflower plants grown on soil were transferred to plastic 15$\mathrm{ml}$ tubes with liquid $0.5 \times$ Murashige and Skoog culture medium supplemented with ACC, JA, IAA, ABA, GA 3 , or SA in the concentrations specified in the figure. Then, tubes with the plants were incubated in growth chamber for $2 \mathrm{~h}$. After that, the cotyledons were harvested and frozen for subsequent analysis. Plants used as controls were treated in the same way, sowing them in $0.5 \times$ Murashige and Skoog liquid culture medium without supplementation.

\section{Number of replicates, number of experiments, and statistical analysis}

To compare the effects of the applied treatments, normality and homogeneity of variances were checked by a Shapiro-Wilk's test and Bartlett's test, respectively. Thereafter, one-way analysis of variance (ANOVA) was done followed by a Fisher LSD post hoc test. To evaluate the RT-qPCR results from assays done to validate microarray results, homogeneity of variances were checked by an $F$ test, and the Student's $t$ test was conducted thereafter. Student's $t$ test was also used to compare enzymatic activities between WT and each transgenic lineage, and a correction applied for multiple $t$ test 
comparison (Benjamini and Yekutieli 2001). Values are mean \pm SD of at least three biological replicates. At least two independent experiments were performed. Details are indicated in the respective figure legends.

\section{Results}

The overexpression of HaWRKY1O in sunflower leaf disks represses many genes involved in primary metabolism

To get information about the role of HaWRKY10 in sunflower, microarray experiments were conducted using a chip previously constructed (see "Materials and methods"). Total RNA isolated from transiently transformed sunflower leaf disks overexpressing HaWRKY10 (HaWRKY10 OX) or with an empty vector as control was used as probes. One hundred and forty-six genes showed significant changes (36\% up, $63 \%$ down) in HaWRKY10 OX. Among them, 109 were putatively identified according to Genbank, and 97 were annotated with one or more gene ontology (GO) terms (Fig. 1 a and Supplementary Table S2). In the first instance, differential expressed genes were mainly annotated by biological processes level 3, with primary metabolic process as the most represented GO considering the node score, a parameter related to the annotation stringency (Fig. 1 b, c; Supplementary Fig. S1). The majority of the sequences was downregulated in HaWRKY10 OX leaf disks and belonged to the "children" GO terms that enroll carbohydrate and protein metabolic processes (Fig. 1d). The annotation was extended according to the Blast2GO pipeline, by adding implicit terms and merging the InterProScan results. As a consequence, the assignment was enriched with new "children" GO terms belonging to the biological process category, chiefly born from the primary metabolic process. Representatives of the new enriched category appeared the specific sucrose and starch metabolic processes, and different amino acids metabolic processes (Fig. 1 e, f).

\section{Fig. 1}

Annotation of microarray results. a Microarray summary. Annotation of the genes differentially regulated in $H a W R K Y 10 \mathrm{OX}$ according to the results of the Blast2GO package pipeline. b Total annotations by GO level and GO categories $(P, F$, and $C) . P$ biological process, $F$ molecular function, $C$ cellular component. c Sequence annotation distribution by the enriched GO level 3 . 
Dark gray bar indicates the GO with highest node score. d Sequence annotation distribution by primary metabolic process children GO terms, according to the node score (light gray bars) and direction of the regulation (black bars, up; white bars, down). e, f Sequence annotation distribution by more specific primary metabolic process children GO terms. In $\mathbf{c}-\mathbf{f}$, a node score $=3$ was used as cutoff 
(a)

\begin{tabular}{l|r|}
\multicolumn{1}{|c|}{ PARAMETERS } & VALUES \\
\hline up-regulated sequences $(\# \mid \%)$ & $52 \mid 35.6$ \\
down-regulated sequences (\#|\%) & $94 \mid 64.4$ \\
sequence length range $(\mathrm{bp})$ & $100-2035$ \\
sequence length average (bp) & 704 \\
identified sequences (\#|\%) & $109 \mid 74.7$ \\
sequences with GO assignation (\#|\%) & $97 \mid 66.4$ \\
\hline
\end{tabular}

(b)

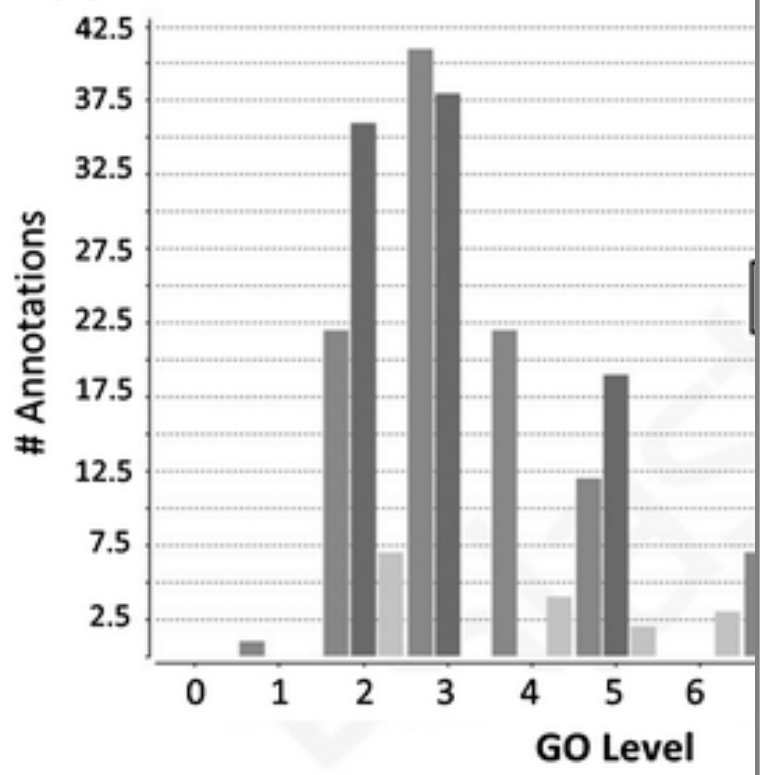

(c)

GO Level 3

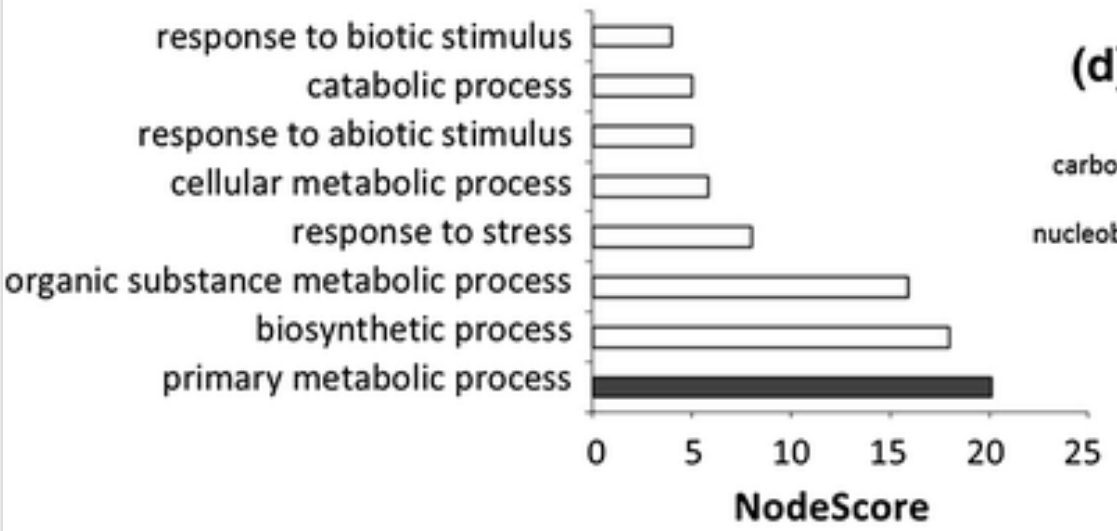

(d)

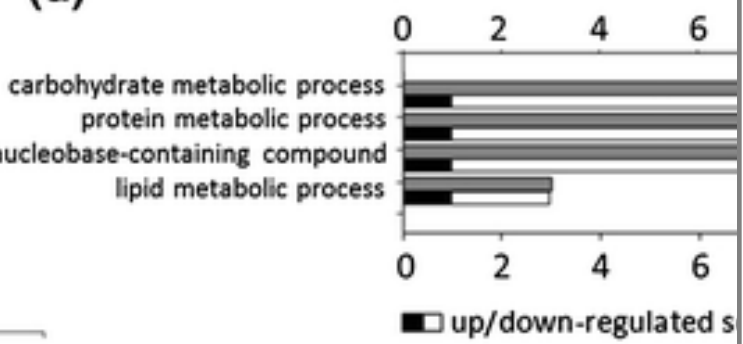

(e)

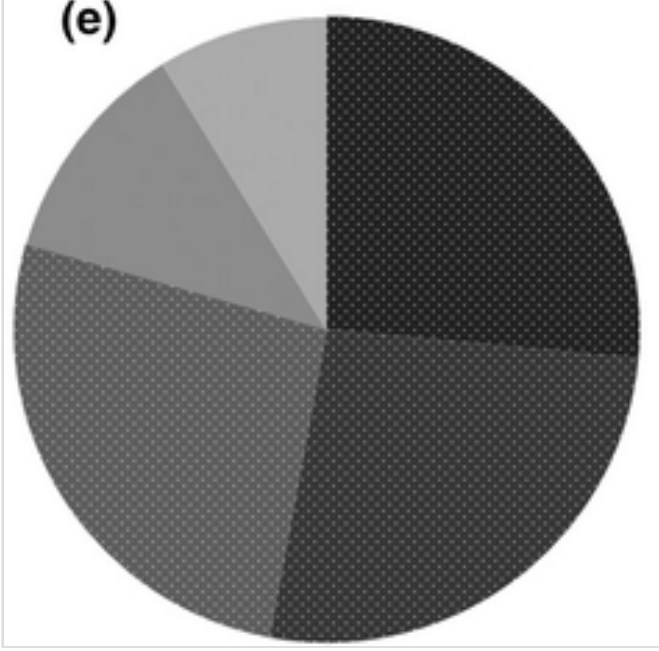

a sucrose metabolic process

z starch metabolic process

a cellular amino acid metabolic process

nucleotide metabolic process

protein phosphorylation (f)

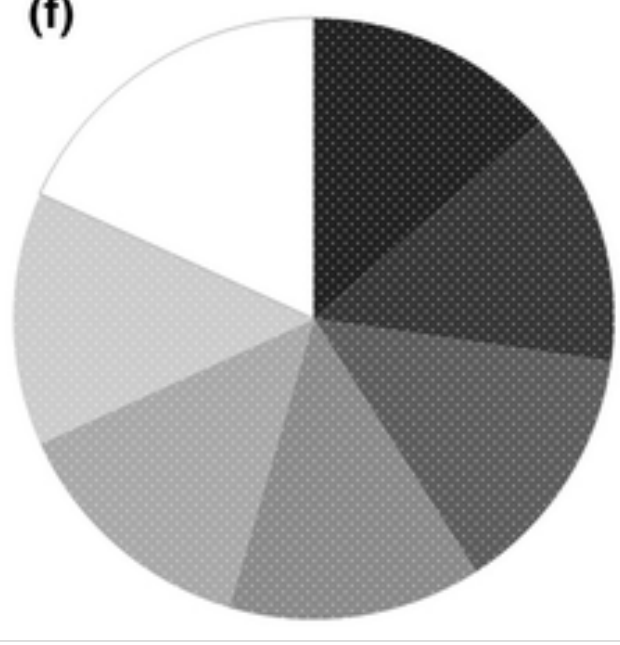

On the other hand, $71 \%$ of the 146 differentially regulated genes retrieved a putative orthologue in A. thaliana (Supplementary Table S3). The differentially expressed genes were put in the context of the potential 
metabolic pathways and other processes in which they could be involved, and then hierarchically organized using SCAVENGER and Pageman modules in Mapman (Supplementary Fig. S2). This analysis also revealed coordinated changes in sets of genes differentially repressed that appeared associated with carbohydrate catabolism (starch consumption and glycolysis) and amino acids synthesis (aspartate family). Conversely, the expression of a gene encoding a putative trehalose-6-phosphatase was slightly induced, which supports a decrease in trehalose-6-phosphate, a metabolite that influences (in a parallel manner) relative amounts of starch and sucrose accumulated (Lunn et al. 2014 ). The results of the array were validated by RT-qPCR in newly transformed sunflower leaf disks samples and superimposed to the Mapman overview (Supplementary Fig. S2). According to this first approach, HaWRKY10 could somehow be involved in the regulation of primary metabolism, mainly of carbohydrates.

\section{HaWRKY1O has expression peaks during germination and post-germination growth of sunflower seeds}

To determine in which tissues and developmental stages HaWRKY10 are expressed, transcript levels were evaluated. HaWRKY10 expression levels were measured in the sunflower vegetative stage, including distinct organs and hormonal treatments. No significant expression peak was observed in different experiments with 7- and 14-day-old plants maintained under standard conditions or in 7-day-old plants exposed to hormonal or stress (abiotic) factors (Supplementary Fig. S3). Conversely, a significant expression peak was detected in dry seeds compared with the levels measured $24 \mathrm{~h}$ after imbibition (h.a.i.). This observation led us to collect time course gene expression data during the seed imbibition process. HaWRKY10 expression peaked 9 h.a.i. in sunflower seeds and, thereafter, significantly decreased (Fig. 2a). Moreover, ABA and GAs regulated HaWRKY10 expression at 9 h.a.i. repressing (tenfold) and inducing (eightfold) it, respectively. Notably, this regulation was organ-specific: both hormones exerted their action in cotyledons, where reserves are stored during dormancy and mobilized during germination, whereas none of them showed any effect in roots (Fig. 2 b). To understand whether HaWRKY10 expression pattern was related to carbon partitioning, samples were gathered up to 24 h.a.i. and different carbon sources were surveyed. As expected, soluble carbohydrates 
(glucose and sucrose) contents decreased quickly, whereas contents of proteins and lipids diminished more slowly. A slight decrease in lipids level occurred at 8 h.a.i., right before the occurrence of an increase in glucose level (1 h later, Fig. 2c). Moreover, lipid and HaWRKY10 transcript levels were inversely correlated ( $\rho=-1, P<0.05$, Fig. 2 c; Supplementary Table S4), in an opposite way of what happened with the other carbon sources.

\section{Fig. 2}

HaWRKY10 expression peaked during the mobilization of seed reserves. a Upper panel HaWRKY10 expression profile during seed germination; lower panel a representative picture of seeds during germination. b Regulation of HaWRKY10 expression in roots and cotyledons by ABA and GA. Transcript levels of HaWRKY10 were quantified by RT-qPCR, normalized with endogenous ACTIN (ACTIN2 and ACTIN8) and, thereafter, with respect to the $0 \mathrm{~h}$ (dry seed) value (a) or the root control values (b), arbitrarily assigned a value of one. c Carbon-partitioning in seeds during germination. Samples were related to the highest value measured, arbitrarily assigned a value of $100 \%$. The initial absolute values $(3 \mathrm{~h})$ in $\mu \mathrm{g} / \mathrm{mg}$ of tissue were: 0.42 for glucose, 14.1 for sucrose, 1.51 for starch, 687 for lipids, and 75 for proteins. The HaWRKY10 expression profile was superimposed (dashed line) to easily visualize the inverse correlation with the lipid expression profile. Arrows point the peak of HaWRKY10 expression. Error bars mean standard deviations from biological replicates. Four biological replicates were done for one out of every three experiments. For the statistical analysis, an ANOVA followed by a Fisher LSD post hoc test was done. Different letters mean significant differences with $P<0.05$ 

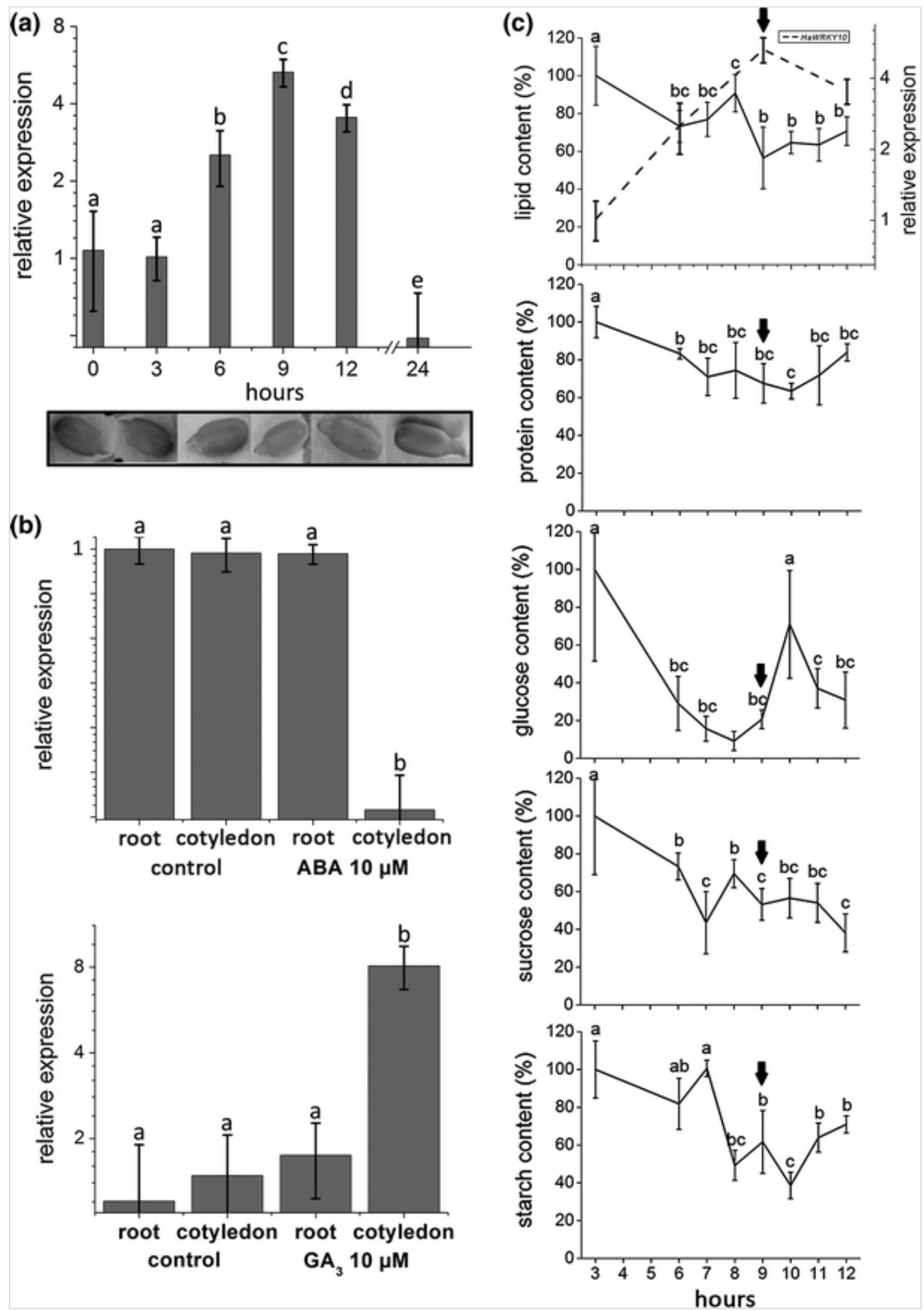
Time course profiles were also assessed during post-germination growth, when lipid consumption is relevant to the lineage establishment. HaWRKY10 was strongly upregulated ( $\sim 128$-fold) by the 4 th day after germination, and its expression continuously increased until the 6th day (Fig. 3 a). Lipid decrease was remarkably high (the final level achieving around $25 \%$ of the initial value) and also inversely proportional to the gene upregulation ( $\rho=0.94$, $P<0.05$, Fig. 3 b; Supplementary Table S4). Sucrose level slightly decreased just at the beginning of chlorophyll biosynthesis. There were no variations in starch levels during the first days of growth, whereas glucose level rose probably nourished by lipid consumption and the incipient photosynthesis (Fig. 3 b).

\section{Fig. 3}

HaWRKY10 expression peaked during the mobilization of seedling carbon sources. a HaWRKY10 expression profile during seedling establishment. Transcript levels of HaWRKY10 were quantified by RT-qPCR, normalized with endogenous ACTIN (ACTIN2 and ACTIN8) and, thereafter, with respect to the value measured the third day, arbitrarily assigned a value of one. b Carbonpartitioning and chlorophyll production during seedling establishment. Samples were related to the highest value measured, arbitrarily assigned a value of $100 \%$. The initial absolute values ( 1 day) in $\mu \mathrm{g} / \mathrm{mg}$ of tissue were: 0.49 for glucose, 2.37 for sucrose, 0.29 for starch, 373 for lipids, and 0.00 for chlorophyll. The HaWRKY10 expression profile was superimposed (dashed line) to easily visualize the inverse correlation with the lipid expression profile. Error bars mean standard deviations from biological replicates. Four biological replicates were done for each experiment of a total of two. For the statistical analysis, an ANOVA followed by a Fisher LSD post hoc test was done. Different letters mean significant differences with $P<0.05$ 

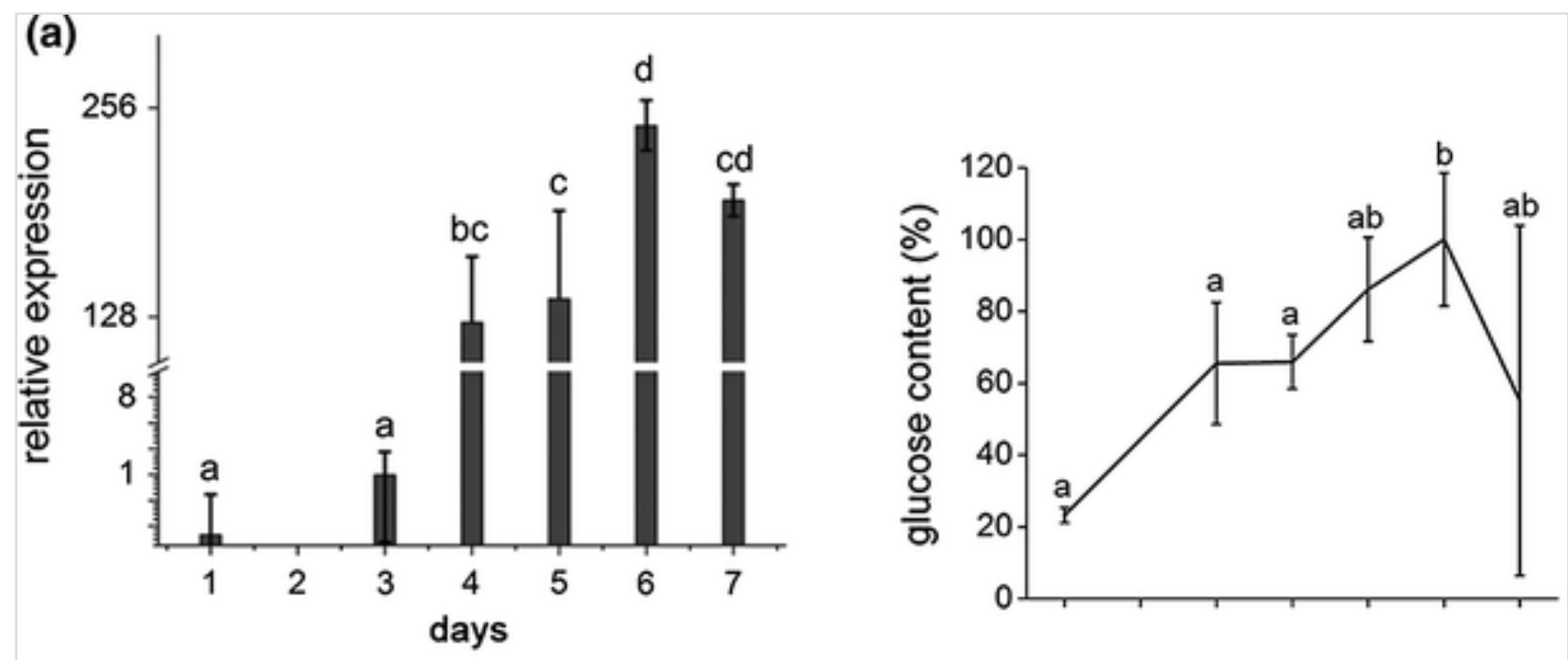

(b)
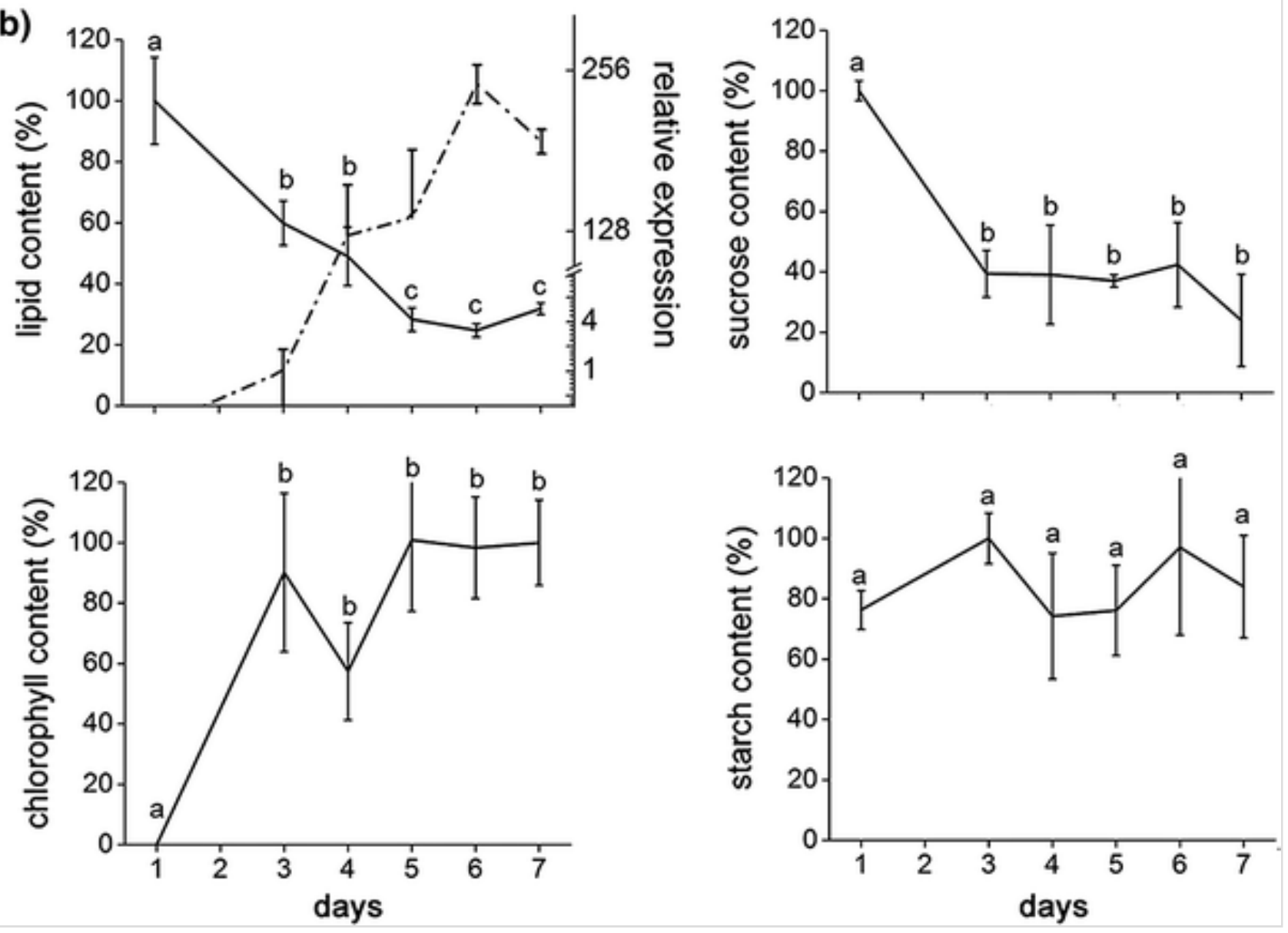

The results of the time course expression profiles of HaWRKY10 and its hormonal regulation suggested that HaWRKY10 is involved in the mobilization of seed reserve compounds during germination and postgermination growth. Revisiting the microarray results, it was possible to establish that a great proportion (61\%) of the differential expressed genes is also expressed in sunflower seeds. In a similar way, a high percentage of the putative orthologues of sunflower genes in Arabidopsis (79\%) was also 
expressed in dry or imbibed seeds (Supplementary Table S2).

\section{Lipid and carbohydrate partitioning differ between Arabidopsis 35S:HaWRKY1O and wild-type seeds and seedlings}

Several Arabidopsis transgenic lineages with constitutive expression of the HaWRKY10 were obtained to study the phenotypic consequences of the transgene ectopic expression, and three of them, exhibiting high expression levels, were chosen for further analyses (Supplementary Fig. S4). Seeds of transgenic and control plants did not differ in color, size, or germination rate; then, seeds were analyzed for their carbon reserves contents after imbibition. Samples were taken at 0 and 48 h.a.i. (Fig. 4), before the photoautotrophic growth became dominant and after the reserves sources had been mobilized. At zero time, lipids and proteins were higher (around 5 and $12 \%$, respectively) in transgenic seeds than in controls, whereas carbohydrates (sucrose and starch) were lower ( $\sim 25$ and $60 \%$, respectively). Because there is an increased interest in improving not only the oil content but also the oil quality of oilseed crops, both oleosin profiles and relative percentages of fatty acids were evaluated. Oleosin proteins are involved in determining oil body's size, which is relevant for the total lipid content. Fatty acid diversity and abundance (e.g., oleic acid quantity) are parameters of oil quality. Our results indicated that oleosin and fatty acid profiles were similar in all the genotypes (Supplementary Fig. S5). Noteworthy, 48 h.a.i. lipids were the main source that was mobilized, and carbohydrates (sucrose and starch) were preserved in transgenic seedlings compared with wild type (WT). In fact, starch level showed a slight increase. Proteins also diminished in all the genotypes, although to a higher extent in the WT than in the transgenics (Fig. 4).

\section{Fig. 4}

Carbon-partitioning in transgenic 35S:HaWRKY10 Arabidopsis seeds compared with WT during germination. Samples were related to the WT value arbitrarily assigned a value of $100 \%$. The initial WT absolute values in $\mu \mathrm{g} / \mathrm{mg}$ of tissue were: 0.46 for glucose, 0.63 for sucrose, 0.06 for starch, 432 for lipids, and 133 for proteins. W10-A, W10-B, and W10-C: three independent transgenic 35S:HaWRKY10 lines. Error bars mean standard deviations from biological replicates. Two experiments were conducted, and three biological replicates 
were done for each one. For the statistical analysis, an ANOVA followed by a Fisher LSD post hoc test was done. Different letters mean significant differences with $P<0.05$
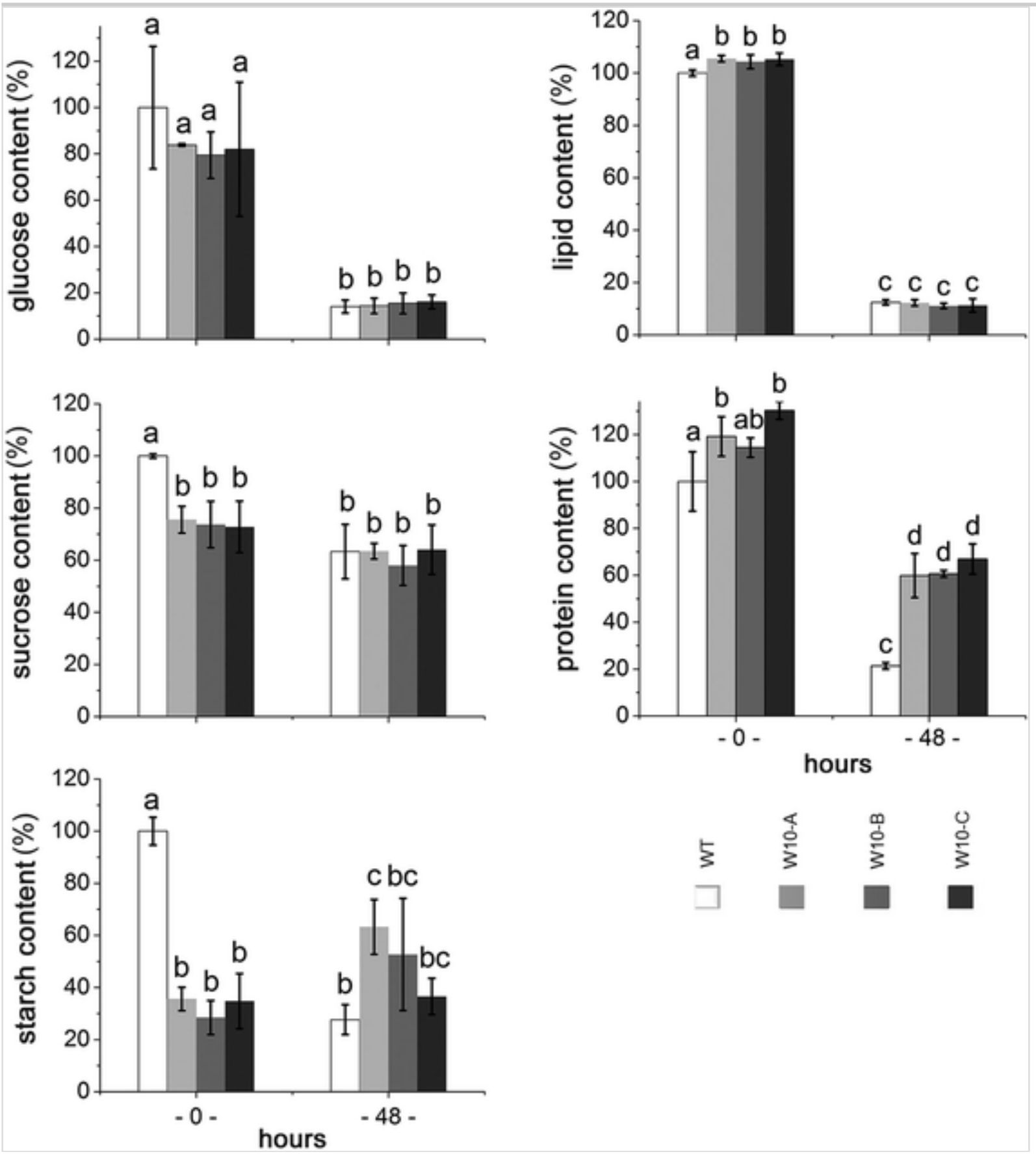

Transgenic 35S:HaWRKY1O seeds exhibit increased gluconeogenesis compared with WT during germination

To evaluate whether the lipid decrease in transgenic seedlings was concomitant with an increase in gluconeogenesis, the expression levels and activities of enzymes playing main roles in gluconeogenesis were assessed at 
36 h.a.i., and a few hours before differences in reserves content were detected between genotypes. SDP1 transcripts were increased in the three transgenic lines compared with the WT (Fig. $5 \mathrm{a}$ ). The activities of PEPCK and fructose 1,6-biphosphate phosphatase (FBPase) were also increased in the same sense (Fig. 5 b). Considering the apparent lower consumption of sucrose in transgenic lines in comparison with the WT, sucrose synthase (SUS) activity was also measured in the physiological degrading direction; however, it did not show significant changes between the majority of transgenics and WT (Fig. 5b). Altogether, these results indicated that transgenic plants showed a differentially stimulated gluconeogenesis compared with WT.

\section{Fig. 5}

Gene expression and enzymes activity in transgenic 35S:HaWRKY10 versus WT Arabidopsis seeds at 36 h.a.i. a SDPl gene expression. b PEPCK, FBPase, and SUS cleaving activities. W10-A, W10-B, and W10-C: three independent transgenic 35S:HaWRKY10 lines. Error bars mean standard deviations from the replicates used in each of two experiments done for each enzyme activity assay. Multiple $t$ tests were done with corrected thresholds. Different letters mean significant differences with $P<0.05$ (except in $b^{*}$ where the threshold increase up to 0.066 ) 
(a)

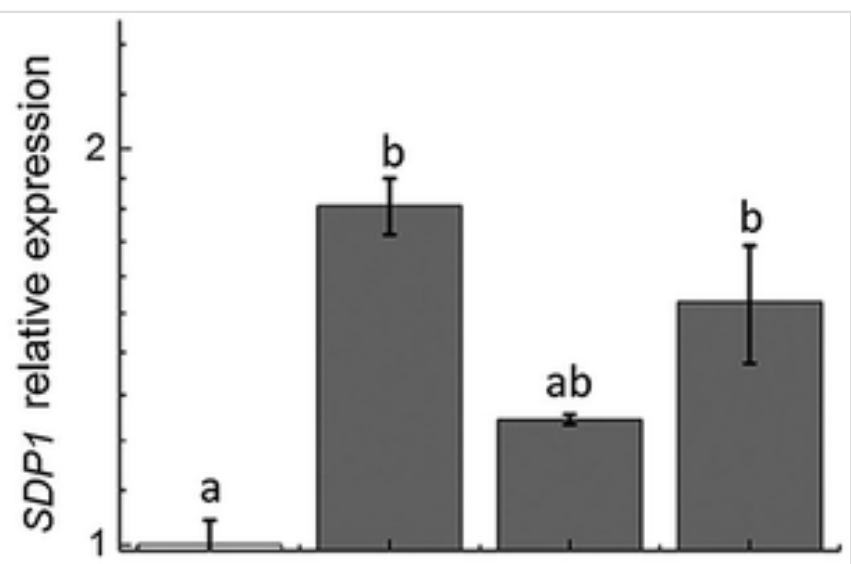

(b)
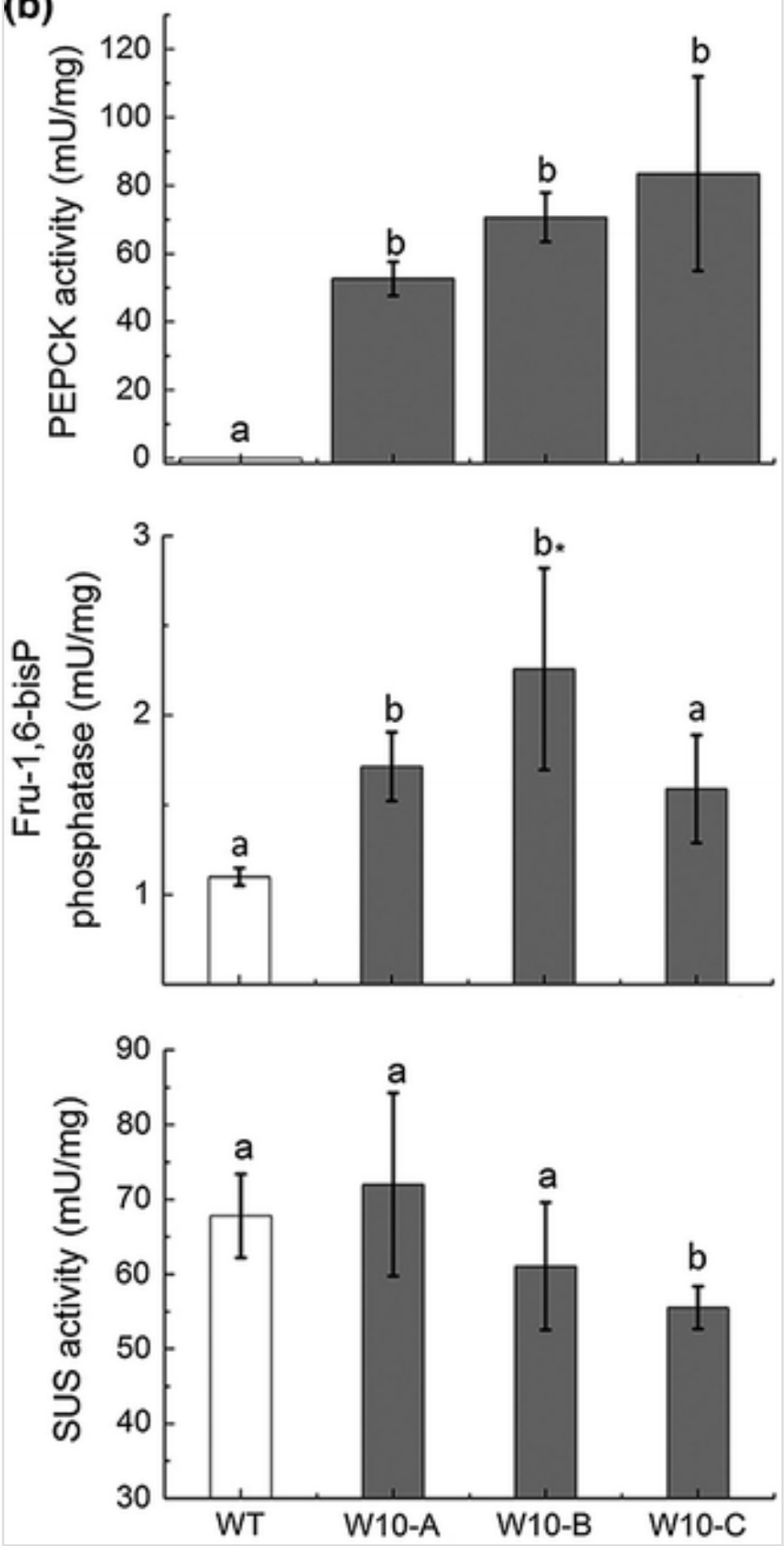
ABA and GA signaling and sensitivity are altered in transgenic 35S:HaWRKY1O seeds during germination respective to the $\mathrm{WT}$

To elucidate how the ABA and GAs transduction pathways are involved in the differential carbon mobilization, expression of genes related to $\mathrm{ABA}$ synthesis ( $A B A 2$ ), ABA negative and positive signaling ( $A B I 1$ and $A B I 4$, respectively), GAs inactivation (GA2OX1), and GAs activation (GA3OX1 and $G A 20 O X 3$ ) was measured (Fig. 6a). The expression of $A B A 2$ and $A B I 1$ genes did not experienced significant changes (data not shown). Conversely, $A B I 4$ mRNA levels, which it is known that decrease during lipolysis, diminished 20 and $35 \%$ more in transgenic plants than in the WT. On the other hand, GA2OX1 and GA20OX3 expression levels were induced and repressed by $\sim 30 \%$, respectively. GA3OX1 expression experienced a trend to reduction, but it was not significant (data not shown). Then, both, ABA positive signaling and GA activation were repressed in transgenic seeds during germination. Considering that ABA/GA balance could be altered in transgenic seeds, the germination rate with and without $\mathrm{ABA}, \mathrm{GA}_{3}$, and a combination of both hormones was evaluated in all the plants. Non-treated seeds of transgenic genotypes germinated with a similar rate as the WT did; however, the former showed a higher sensitivity to ABA (Fig. 6b). In the same sense, post-germination growth was also notably more affected by ABA in transgenic than in the WT seedlings (Fig. 6c). Gibberellins treatment alone had no differential effect on lineages but partially reverted the ABA sensitivity, and observed during germination (Fig. 6d).

Fig. 6

ABA and GA signaling genes and hormonal sensitivity in transgenic 35S:HaWRKY10 Arabidopsis seeds during germination. a Differential expression of genes involved in ABA signal transduction and GA biosynthesis pathways. ABA effect on $\mathbf{b}$ germination and $\mathbf{c}$ post-germination growth, and $\mathbf{d}$ $\mathrm{GA}_{3}$ reversion of the $\mathrm{ABA}$ effect on germination. Germination was measured at $48 \mathrm{~h}$ (controls) and $72 \mathrm{~h}$ (treated). W10-A, W10-B, and W10-C: three independent transgenic 35S:HaWRKY10 lines. Error bars mean standard deviations from biological replicates. Thirty seeds were used per sample. Three biological replicates were done for each experiment of a total of two 
e.Proofing

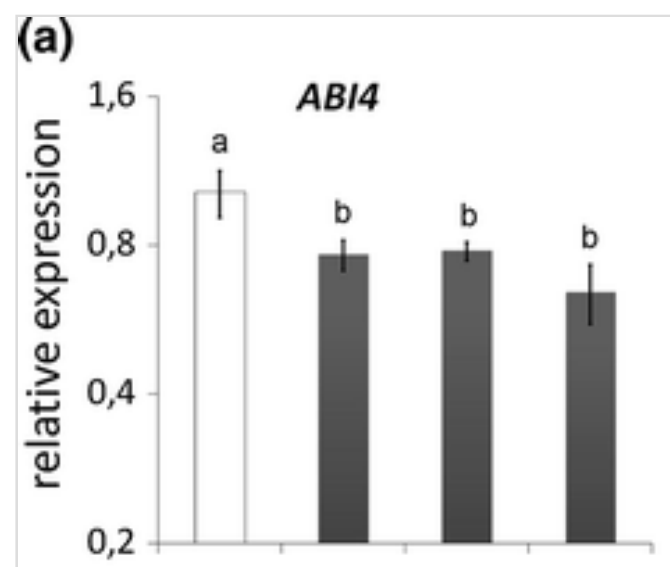

(b) 120
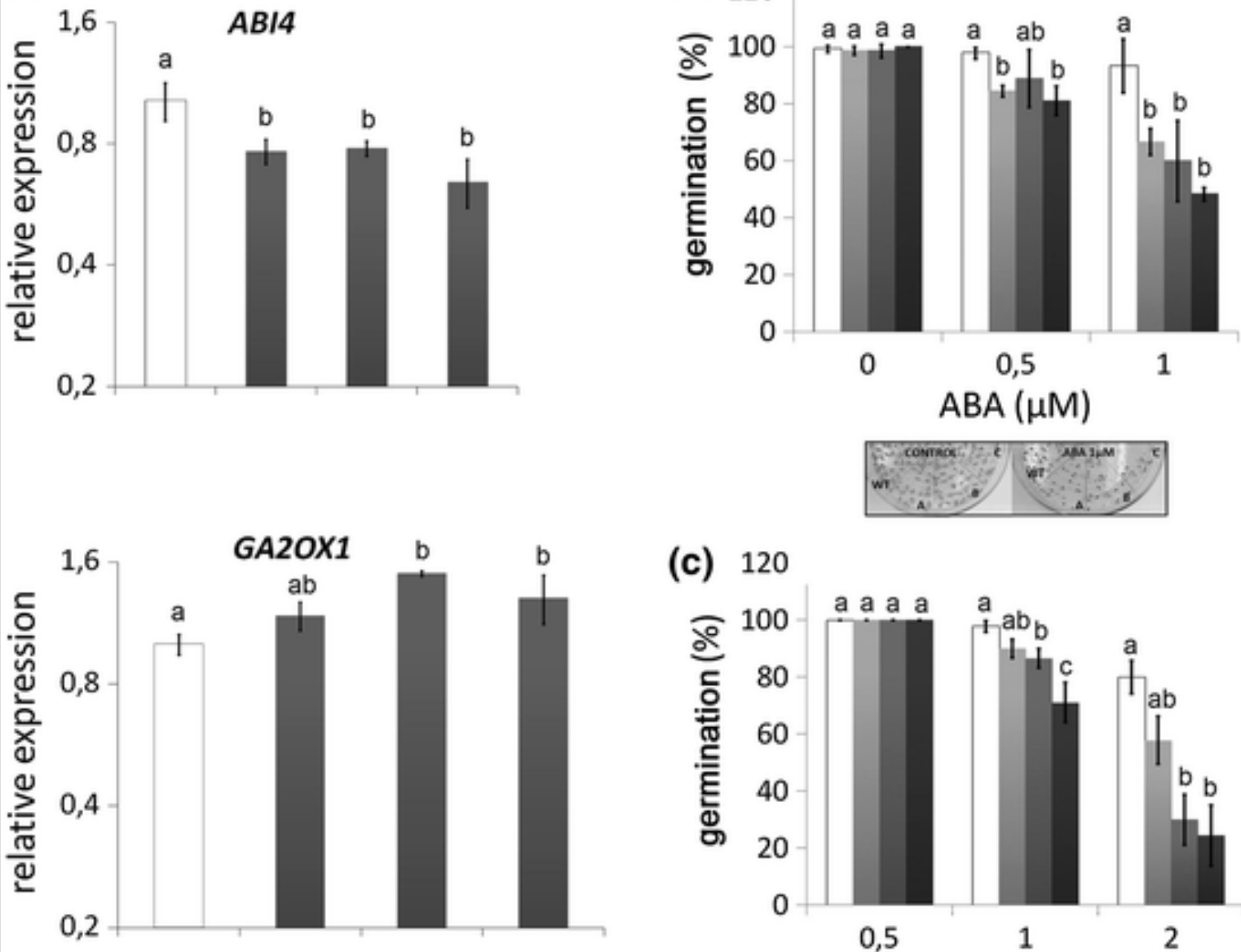

(c) 120
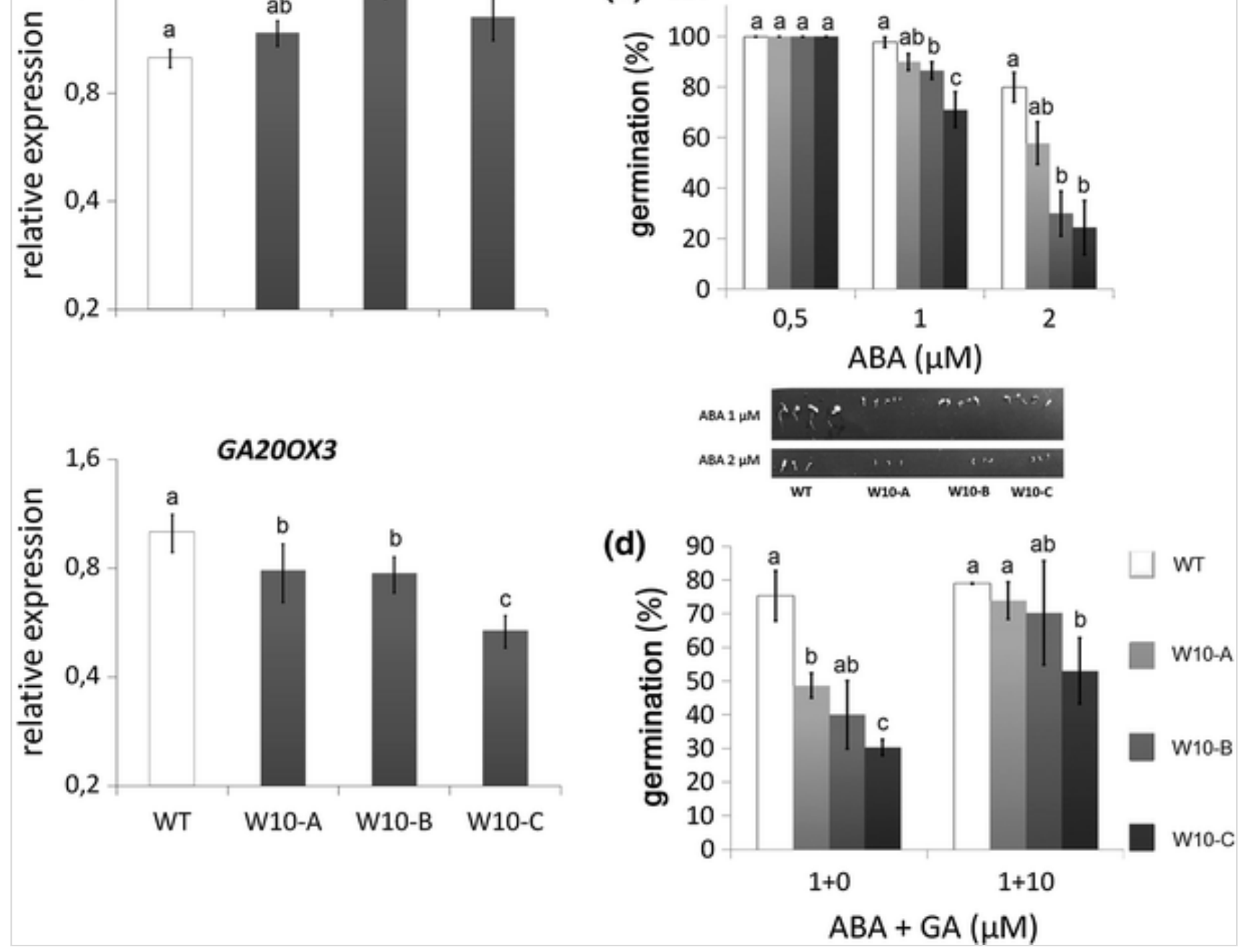

\section{Discussion}

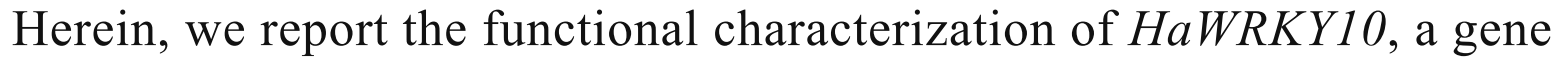
member of the sunflower WRKY TFs family, resolved in the group IId (Giacomelli et al. 2010). The transcriptome analysis of HaWRKY10 
transiently transformed sunflower leaf disks revealed that several genes of the glycolytic and protein degradation pathways were repressed. Moreover, HaWRKY10 expression analysis showed a hormone-dependent regulation in the embryo leaves or cotyledons of imbibed seeds $48 \mathrm{~h}$ after seed imbibition, suggesting a role for this TF in reserves mobilization. A few WRKY TFs of monocotyledons were functionally associated with reserve mobilization so far. For example, SUSIBA2, a TF isolated from barley endosperm tissue, was found to bind in vitro to cis-nucleotide stretches (the sugar responsive SURE elements) in the promoter of isoamylase, an essential enzyme in amylopectin synthesis (Sun et al. 2003 ). ABF1 and ABF2 from wild oat and the rice OsWRKY51 were also found to bind in vitro to the promoter of the $\alpha$ amylase gene, a key enzyme in the catabolism of starch (Rushton et al. 1995; Xie et al. 2006). OsWRKY51 is a putative orthologue of the sunflower HaWRKY10 (Giacomelli et al. 2010). Also, as a repressor of the $\alpha$-amylase gene, OsWRKY51 was found to act in a synergistic way with another WRKY TF, OsWRKY71 (Xie et al. 2006). A similar function was assigned to HvWRKY38, another member of the barley group IId (Xie et al. 2007). On the other hand, OsWRKY51 and HvWRKY38 were assigned a role in the blockage of the stored carbohydrate mobilization and the germination stunt. Moreover, the expression of both genes was repressed by GAs and induced by ABA in seeds (Mena et al. 2002; Xie et al. 2006, 2007; Zhang et al. 2004). Conversely, HaWRKY10 was induced by GAs and repressed by ABA in sunflower cotyledons, suggesting an opposite function to that described for OsWRKY51 and HvWRKY38. The results also appeared to reflect an increased expression of HaWRKY10, although without a significant peak, in cotyledons and other photosynthetic organs from 14-day-old plants (Supplementary Fig. S3), which could indicate that HaWRKY10 has a role in other stages of the plant life cycle not explored in this work. HaWRKY10 time course expression during both germination and post-germination growths of sunflower seeds correlated with lipid mobilization, which suggested that both processes could be associated. Moreover, without variations in starch levels during the first day of development, glucose rise would be provided by lipid consumption and the incipient photosynthesis. If this was the case, HaWRKY10 would be involved in the mobilization of seed reserve compounds during germination or mainly involved in triggering lipid catabolism in post-germination growth, and gluconeogenesis would increase 
concomitantly with lipid consumption.

As the hormonal dependence of lipid mobilization has been well established in the oilseed species Arabidopsis (Penfield et al. 2004), transgenic Arabidopsis seeds expressing HaWRKY10 were used as a tool to pursue this TF characterization. The constitutive and ectopic expression of the TF did not produce morphological nor phenological changes in Arabidopsis plants. At the same way, no significant changes were observed in leaf area, volume, biomass, or yield of transgenic plants. Then, the phenotypical characterization during germination and post-germination growths was continued. A model of the HaWRKY10 action is schematized in Fig. 7. Differently from WT, transgenic seeds produced more lipids, the majority of the lineages also more proteins, and less carbohydrates (sucrose and starch) than WT as reserves. These results showed a relationship with the detected blockage of lipid and protein degradation pathways observed in microarray experiments with sunflower leaf disks. On the other hand, the general repression found in carbohydrates catabolism in sunflower is also in agreement with the low levels of carbohydrates measured in transgenic Arabidopsis seeds. A putative TPPG was slightly induced according to microarrays and several studies confirmed that there is a parallel correlation between the trehalose 6-phosphate (Tre6P) and sucrose amounts in Arabidopsis through different life stages, and it could be extended to other plant species (Lunn et al. 2014). Then, it is tempting to speculate that a reduced level of Tre6P would be in agreement with the lower levels of sucrose measured. These results suggested that HaWRKY10 could also have a role in seed filling. Moreover, normalized measurements indicated that transgenic seeds appeared to preferentially consume more lipids (around $7 \%$ ) than WT and not to demand sucrose, whose idle excess would nourish the slight starch increment. Protein levels also decreased but preferentially in the WT (the difference is around $15 \%$ ). In a recent study, it was stated that the route of glucose synthesis from pyruvate, a major product from protein breakdown, is also used in Arabidopsis during germination and seedling stablishment (Eastmond et al. 2015). Thus, this route would be less active in the transgenic Arabidopsis seeds expressing HaWRKY10. Hence, an increase in gluconeogenesis in these plants would be expected to consume the mobilized lipids. Gene expression levels and activity of enzymes involved in 
the pathways of lipolysis and gluconeogenesis increased together, producing changes in carbon balance, with significant differences detected 36 h.a.i. This was coincident with the reported coordinated increase in mRNAs and enzyme activities of $\beta$-oxidation (the acyl-CoA oxidase- and 3-ketoacyl-CoA thiolase), glyoxylate (the isocitrate lyase and malate synthase) and gluconeogenesis (the PEPCK) pathways, all visible early and peaking $48 \mathrm{~h}$ after imbibition (Rylott et al. 2001). Concurrently, $A B I 4$ expression was repressed in HaWRKY10 transgenic seeds. Increase in $A B I 4$ expression in dormant Arabidopsis embryo as well as its role as repressor of lipid breakdown and its upregulation by ABA had been previously reported (Penfield et al. 2006). Hence, our observations regarding glycolytic activities and $A B I 4$ expression are in agreement with the increase in lipid mobilization. On the other hand, HaWRKY10 function as transcription factor was tested in an in vivo assay, by transient co-transformation of Nicotiana benthamiana disk leaves with 35S:HaWRKY10 and a construct with a four W-boxes tandem promoter directing the expression of the reporter gene GUS (Supplementary Fig. S6). HaWRKY10 could work as a transcriptional activator, which contributes to support HaWRKY10 as a positive regulator of gene expression and some of the genes whose expression changed in the HaWRKY10 overexpression background as direct targets of it.

\section{Fig. 7}

Proposed model for the role of sunflower HaWRKY10 in seed establishment. The diagram summarizes the proposed interactions between reserve substances, hormones, gene transcripts, enzyme activities, and HaWRKY10 during seeds germination. Hypothetical interactions are distinguished from interactions registered in this work or in the literature through dashed lines. Sharp arrows indicate induction, and blunt arrows indicate repression 


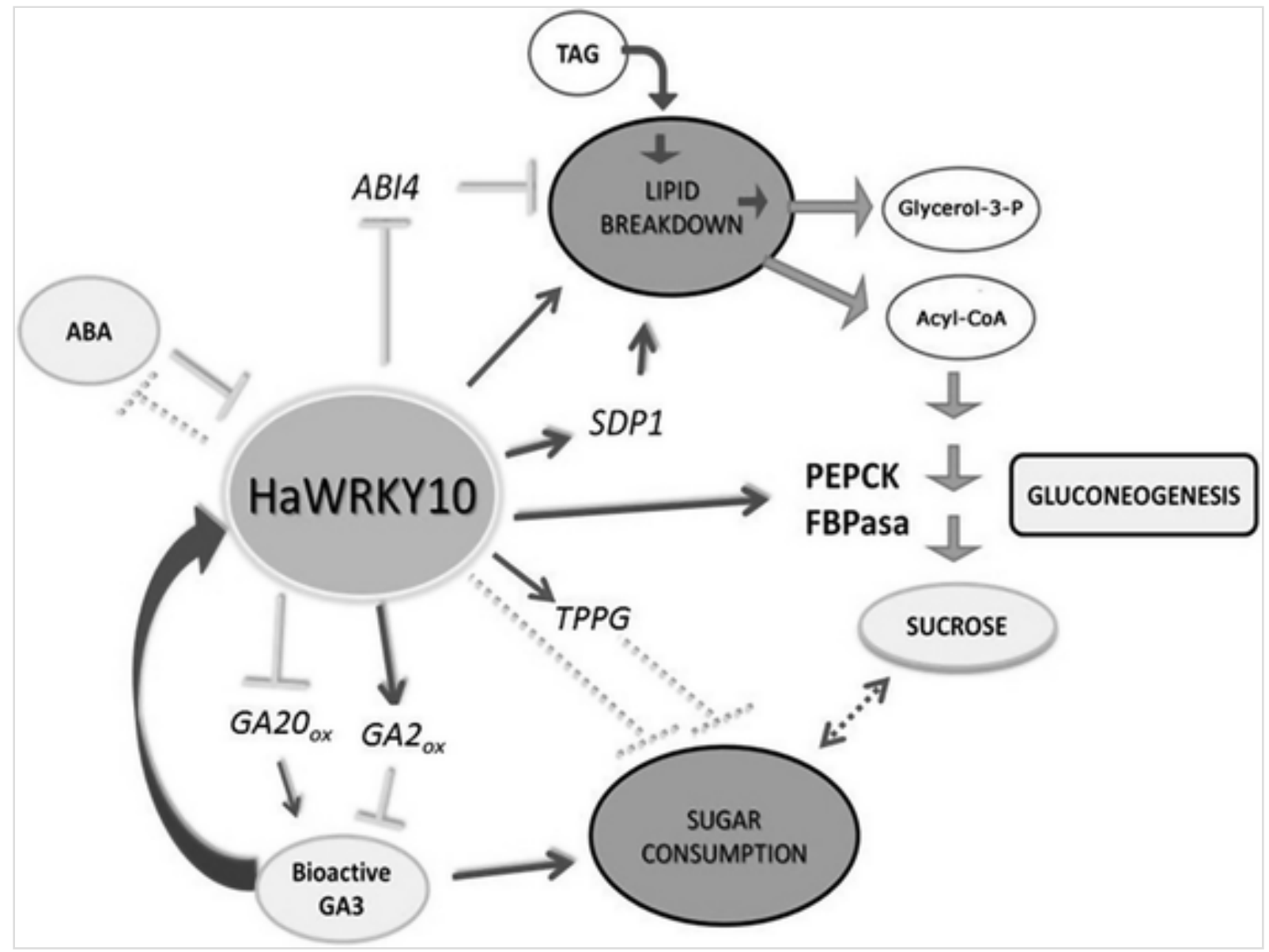

Transgenic HaWRKY10 Arabidopsis seeds were more sensitive to ABA than their controls; however, this enhanced sensitivity was partially rescued by GAs. Studies performed with the dormant model $A$. thaliana ecotype Cvi, indicated that the ABA:GA balance governs the transition between dormant and non-dormant stages (Ali-Rachedi et al. 2004; Cadman et al. 2006). Moreover, the timing of germination in cereals is regulated by the hormone ratio, accompanied by changes in sensitivity to the hormones and affected by environmental factors (Finch-Savage and Leubner-Metzger 2006; and references therein). The differential regulation of $A B I 4, G A 2 O X 1$ and GA20OX3 expression in HaWRKY10 transgenic seeds together with variances in lipid mobilization compared with WT, suggested that the ABA:GA balance and/or the hormone sensitivity are dissimilarly regulated in the transgenics. This suggestion is supported by the partial reversion of the ABA effect on transgenic seeds germination achieved with the external addition of $\mathrm{GA}_{3}$. Moreover, one of the repressed genes identified in the transcriptome analysis encodes a putative orthologue of an Arabidopsis cytochrome P450 (CYP450) gene, only expressed in seeds, CYP76C7 (Supplementary Table S2). Based on the current knowledge, the subfamily CYP76C is exclusive of Brassicaceae; 
therefore, the sunflower gene is probably an orthologue of $C Y P 76 B 1$ from $H$. tuberosus, which has the sequence with highest similarity in the available data from Asteraceae (Hofer et al. 2014). Anyway, several members of the CYP450 superfamily have been involved in the ABA catabolism, which is necessary during dormancy release (Okamoto et al. 2006). The repression of the putative CYP76 member found in sunflower leaf disks is in agreement with the downregulation of CYP76C7 detected in transgenic-HaWRKY10 Arabidopsis seeds by RT-qPCR with specific oligonucleotides (data not shown). On the other hand, the genes related to ABA and GA signaling, whose expression levels changed in Arabidopsis transgenic seedlings ( $A B I 4$, $G A 2 O X 1$, and $G A 20 O X 3$ ) were searched in the sunflower chip and are apparently absent. This conclusion was arrived after searching for them through a bidirectional best hit (BBH) test, a commonly accepted approach to identify putative orthologues (Overbeek et al. 1999; Altenhoff and Dessimoz 2009).

According to the results presented above, HaWRKY10 would be a regulator of seed reserve mobilization during germination and seedling establishment, acting through a mechanism that would involve ABA/GA pathways and gluconeogenesis enhancing.

AQ4

Author contribution statement JR performed the experimental assays, and contributed with the data analysis and figures design. $\mathrm{MH}$ performed part of the experimental assays and figures design. RLC and AAI analyzed data and drafted the MS. KFR designed the study, coordinated the experiments and data analysis, and drafted the manuscript. All the authors read and approved the MS.

\section{Acknowledgments}

This work was supported by Agencia Nacional de Promoción Científica y Tecnológica [ANPCyT, PICT-PAE-37100, PICT-2011-850, PICT-2012-2439], Consejo Nacional de Investigaciones Científicas y Técnicas [CONICET, PIP 2011 11420100100278] and Universidad Nacional del Litoral [UNL, CAID 201150120110100349 and 50120110100399]. JR and MDH are Fellows of CONICET and members of UNL; RLC, AAI and KFR are career members of 
CONICET and UNL.

Compliance with ethical standards

Conflict of interest The authors declare that they have no conflict of interest.

\section{Electronic supplementary material}

Below is the link to the electronic supplementary material.

Supplementary material 1 (DOCX $13 \mathrm{~kb})$

Supplementary material 2 (XLSX $2796 \mathrm{~kb})$

Supplementary Figure S1. Combined GO annotation graph. The sequences differentially regulated in HaWRKY10 OX leaf disks were functional annotated by GO terms and the complete annotation represented at the same time. Left column and transverse bars indicate GO level. GO term orange coloring intensity is proportional to the node score and, then, to the annotation density (EPS $3465 \mathrm{~kb}$ )

Supplementary Figure S2. Mapman overview of selected differential expressed genes in HaWRKY10 OX. Data were extracted from the microarray experiment and validated by RT-qPCR. Red and green boxes represent upregulated and downregulated genes, respectively. Plots represent genes validated by RTqPCR. The expression differences were confirmed in seven out of nine selected genes. Different letters mean significant differences with $P<0.05$. Normalization was made with endogenous ACTIN (ACTIN2 and ACTIN8) and, thereafter, with the control sample (without $H a W R K Y 10$ ), arbitrarily assigned a value of one. W10 and 121 indicate sunflower tissue samples with or without overexpressed $H a W R K Y 10$, respectively (EPS $290 \mathrm{~kb}$ )

Supplementary Figure S3. HaWRKY10 expression in sunflower plants. HaWRKY10 expression was measured in (a) organs of 7- and 14-day-old plants 
and (b) embryo leaves or cotyledons of 7 day-old plants under hormonal treatments. Normalization was made with endogenous ACTIN (ACTIN2 and ACTIN8) and, thereafter, with the $\mathrm{R}$ or the A samples (a, 7- and 14-day-oldplants, respectively) or with the control sample (b), arbitrarily assigned a value of one. $\mathrm{R}=$ root, $\mathrm{H}=$ hypocotyl, $\mathrm{C}=$ cotyledons, $\mathrm{A}=$ shoot apex, $\mathrm{L}=$ leaves; $\mathrm{CTL}=$ control, $\mathrm{ABA}=$ abscisic acid, $\mathrm{ACC}=1$-aminocyclopropane-1carboxylic acid, $\mathrm{GA}_{3}$ : gibberellic acid 3, IAA = indole-3-acetic acid, $\mathrm{JA}=$ methyl-jasmonic acid, $\mathrm{SA}=$ salicylic acid (EPS $1035 \mathrm{~kb})$

Supplementary Figure S4. Expression levels of HaWRKY10 in several independent homozygous transgenic lines of Arabidopsis. Transcript levels of HaWRKY10 were quantified by RT-qPCR, normalized with endogenous ACTIN (ACTIN2 and ACTIN8) and, thereafter, with respect to the lower expression line arbitrarily assigned a value of one. Asterisks indicate the three lines chosen to continue the study, named W10-A, W10-B, and W10-C, respectively. Four biological replicates were done for each experiment of a total of two (EPS $686 \mathrm{~kb})$

Supplementary Figure S5. Fatty acids profile (a) and oleosins (b) in HaWRKY10 Arabidopsis transgenic seeds versus WT. SAT: saturated, UNSAT: unsaturated with 1, 2, or 3 double bonds between the carbon atoms as indicated. OLEO: oleosin 1, 2, or 4 as indicated. Lane named MM corresponds to molecular mass markers, with sizes specified in kDa. W10-A, W10-B, and W10-C: three independent transgenic 35S:HaWRKY10 lines (EPS $289 \mathrm{~kb}$ )

Supplementary Figure S6. HaWRKY10 induces the expression of a reporter gene through the binding to a promoter with cis-acting $\mathrm{W}$ box elements. Nicotiana benthamiana leaf disks were infiltrated with a A. tumefaciens expressing:GUS (negative control), pOs:GUS, 35S:GUS (positive control), pOs:GUS plus 35S:TF1, pOs:GUS plus 35S:TF2, pOs:GUS plus 35S:HaWRKY10. GUS: betaglucuronidase cDNA, pOs: promoter of the Os03g16950 rice gene with five $\mathrm{W}$ box elements, 35S: constitutive CaMV promoter, $T F 1$ : a non-W box recognizing TF cDNA, TF2: a W box recognizing TF cDNA (EPS $496 \mathrm{~kb}$ ) 


\section{References}

Al-Shahrour F, Minguez P, Vaquerizas JM, Conde L, Dopazo J (2005)

BABELOMICS: a suite of web tools for functional annotation and analysis of groups of genes in high-throughput experiments. Nucleic Acids Res 33:W460-W464

Ali-Rachedi S, Bouinot D, Wagner MH, Bonnet M, Sotta B, Grappin P, Jullien M (2004) Changes in endogenous abscisic acid levels during dormancy release and maintenance of mature seeds: studies with the Cape Verde Islands ecotype, the dormant model of Arabidopsis thaliana. Planta 219:479-488

Altenhoff AM, Dessimoz C (2009) Phylogenetic and functional assessment of orthologs inference projects and methods. PLoS Comput Biol 5:e1000262

Balasaraswathi R, Sadasivam S (1997) Changes in oil, sugars and nitrogenous components during germination of sunflower seeds, Helianthus annuus. Plant Foods Hum Nutr 51:71-77

Benjamini Y, Yekutieli D (2001) The control of the False Discovery Rate in multiple testing under dependency. Ann Stat 29:1165-1188

Bewley JD, Black M (1994) Seeds. Physiology of development and germination. Springer, New York

Bewley JD, Bradford K, Hilhorst H, Nonogaki H (2013) Seeds.

Physiology of development, germination and dormancy. Plenum Press, New York

Bradford MM (1976) A rapid and sensitive method for the quantitation of microgram quantities of protein utilizing the principle of protein-dye binding. Anal Biochem 72:248-254 
Cadman CS, Toorop PE, Hilhorst HW, Finch-Savage WE (2006) Gene expression profiles of Arabidopsis Cvi seeds during dormancy cycling indicate a common underlying dormancy control mechanism. Plant J 46:805-822

Capella M, Re DA, Arce AL, Chan RL (2014) Plant homeodomain-leucine zipper I transcription factors exhibit different functional AHA motifs that selectively interact with TBP or/and TFIIB. Plant Cell Rep 33:955-967

Clough SJ, Bent AF (1998) Floral dip: a simplified method for Agrobacterium-mediated transformation of Arabidopsis thaliana. Plant $\mathrm{J}$ $16: 735-743$

Conesa A, Gotz S, Garcia-Gomez JM, Terol J, Talon M, Robles M (2005) Blast2GO: a universal tool for annotation, visualization and analysis in functional genomics research. Bioinformatics 21:3674-3676

Corbineau F, Xia Q, Bailly C, El-Maarouf-Bouteau H (2014) Ethylene, a key factor in the regulation of seed dormancy. Front Plant Sci 5:539

Cornah JE, Germain V, Ward JL, Beale MH, Smith SM (2004) Lipid utilization, gluconeogenesis, and seedling growth in Arabidopsis mutants lacking the glyoxylate cycle enzyme malate synthase. J Biol Chem 279:42916-42923

Chen H, Lai Z, Shi J, Xiao Y, Chen Z, Xu X (2010) Roles of Arabidopsis WRKY18, WRKY40 and WRKY60 transcription factors in plant responses to abscisic acid and abiotic stress. BMC Plant Biol 10:281

Ding ZJ, Yan JY, Li GX, Wu ZC, Zhang SQ, Zheng SJ (2014) WRKY41 controls Arabidopsis seed dormancy via direct regulation of ABI3 transcript levels not downstream of ABA. Plant J 79:810-823

Eastmond PJ (2006) SUGAR-DEPENDENT1 encodes a patatin domain triacylglycerol lipase that initiates storage oil breakdown in germinating Arabidopsis seeds. Plant Cell 18:665-675 
Eastmond PJ, Astley HM, Parsley K, Aubry S, Williams BP, Menard GN, Craddock CP, Nunes-Nesi A, Fernie AR, Hibberd JM (2015) Arabidopsis uses two gluconeogenic gateways for organic acids to fuel seedling establishment. Nat Commun 6:6659

Eastmond PJ, Graham IA (2001) Re-examining the role of the glyoxylate cycle in oilseeds. Trends Plant Sci 6:72-78

Eckardt NA (2005) Peroxisomal citrate synthase provides exit route from fatty acid metabolism in oilseeds. Plant Cell 17:3

Eulgem T, Somssich IE (2007) Networks of WRKY transcription factors in defense signaling. Curr Opin Plant Biol 10:366-371

Fernandez P, Fernandez P, Soria M, Blesa D, DiRienzo J, Moschen S, Rivarola M, Clavijo BJ, Gonzalez S, Peluffo L, Príncipi D, Dosio G, Aguirrezabal L, García-García F, Conesa A, Hopp E, Dopazo J, Heinz RA, Paniego N (2012) Development, characterization and experimental validation of a cultivated sunflower (Helianthus annuus L.) gene expression oligonucleotide microarray. PLoS One 7:e45899

Finch-Savage WE, Leubner-Metzger G (2006) Seed dormancy and the control of germination. New Phytol 171:501-523

Frohman MA, Dush MK, Martin GR (1988) Rapid production of fulllength cDNAs from rare transcripts: amplification using a single genespecific oligonucleotide primer. Proc Natl Acad Sci USA 85:8998-9002

Fusari C, Demonte AM, Figueroa CM, Aleanzi M, Iglesias AA (2006) A colorimetric method for the assay of ADP-glucose pyrophosphorylase. Anal Biochem 352:145-147

Giacomelli JI, Ribichich KF, Dezar CA, Chan RL (2010) Expression analyses indicate the involvement of sunflower WRKY transcription factors in stress responses, and phylogenetic reconstructions reveal the existence of a novel clade in the Asteraceae. Plant Sci 178:398-410 
Gocal GF et al (2001) GAMYB-like genes, flowering, and gibberellin signaling in Arabidopsis. Plant Physiol 127:1682-1693

Gómez-Cadenas A, Zentalla R, Walker-Simmons M, Ho T-HD (2001) Gibberellin/abscisic acid antagonism in barley aleurone cells: site of action of the protein kinase PKABA1 in relation to gibberellin signaling molecules. Plant Cell 13:3

Green F, Clausen CA, Highley TL (1989) Adaptation of the NelsonSomogyi reducing-sugar assay to a microassay using microtiter plates. Anal Biochem 182:3

Gubler F, Chandler PM, White RG, Llewellyn DJ, Jacobsen JV (2002) Gibberellin signaling in barley aleurone cells. Control of SLN1 and GAMYB expression. Plant Physiol 129:191-200

Gubler F, Kalla R, Roberts JK, Jacobsen JV (1995) Gibberellin-regulated expression of a myb gene in barley aleurone cells: evidence for Myb transactivation of a high-pI alpha-amylase gene promoter. Plant Cell $7: 1879-1891$

Gubler F, Millar AA, Jacobsen JV (2005) Dormancy release, ABA and pre-harvest sprouting. Curr Opin Plant Biol 8:183-187

Hoekema A, Hirsch PR, Hooykaas PJJ, Schilperoort RA (1983) A binary plant vector strategy based on separation of vir- and T-region of the Agrobacterium tumefaciens Ti-plasmid. Nature 303:2

Hofer R et al (2014) Dual function of the cytochrome P450 CYP76 family from Arabidopsis thaliana in the metabolism of monoterpenols and phenylurea herbicides. Plant Physiol 166:1149-1161

Jiang T, Zhang XF, Wang XF, Zhang DP (2011) Arabidopsis 3-ketoacylCoA thiolase-2 (KAT2), an enzyme of fatty acid beta-oxidation, is involved in ABA signal transduction. Plant Cell Physiol 52:528-538

Kelly AA, Quettier AL, Shaw E, Eastmond PJ (2011) Seed storage oil 
mobilization is important but not essential for germination or seedling establishment in Arabidopsis. Plant Physiol 157:866-875

Laemmli UK (1970) Cleavage of structural proteins during the assembly of the head of bacteriophage T4. Nature 227:680-685

Linkies A, Leubner-Metzger G (2012) Beyond gibberellins and abscisic acid: how ethylene and jasmonates control seed germination. Plant Cell Rep 31:253-270

Lunn JE, Delorge I, Figueroa CM, Van Dijck P, Stitt M (2014) Trehalose metabolism in plants. Plant J 79:544-567

Malone S, Chen ZH, Bahrami AR, Walker RP, Gray JE, Leegood RC (2007) Phosphoenolpyruvate carboxykinase in Arabidopsis: changes in gene expression, protein and activity during vegetative and reproductive development. Plant Cell Physiol 48:441-450

Manavella PA, Chan RL (2009) Transient transformation of sunflower leaf discs via an Agrobacterium-mediated method: applications for gene expression and silencing studies. Nat Protoc 4:1699-1707

Manavella PA, Dezar CA, Ariel FD, Drincovich MF, Chan RL (2008) The sunflower HD-Zip transcription factor HAHB4 is up-regulated in darkness, reducing the transcription of photosynthesis-related genes. J Exp Bot 59:3143-3155

Mena M, Cejudo FJ, Isabel-Lamoneda I, Carbonero P (2002) A role for the DOF transcription factor BPBF in the regulation of gibberellinresponsive genes in barley aleurone. Plant Physiol 130:111-119

Meyer K, Stecca KL, Ewell-Hicks K, Allen SM, Everard JD (2012) Oil and protein accumulation in developing seeds is influenced by the expression of a cytosolic pyrophosphatase in Arabidopsis. Plant Physiol $159: 1221-1234$

Mi H, Muruganujan A, Thomas PD (2013) PANTHER in 2013: modeling 
the evolution of gene function, and other gene attributes, in the context of phylogenetic trees. Nucleic Acids Res 41:D377-D386

Moschen S et al (2016) Integrating transcriptomic and metabolomic analysis to understand natural leaf senescence in sunflower. Plant Biotechnol J 14:719-734

Nonogaki H (2008) Repression of transcription factors by microRNA during seed germination and postgerminaiton: another level of molecular repression in seeds. Plant Signal Behav 3:65-67

Okamoto $\mathrm{M}$ et al (2006) CYP707A1 and CYP707A2, which encode abscisic acid 8'-hydroxylases, are indispensable for proper control of seed dormancy and germination in Arabidopsis. Plant Physiol 141:97-107

Overbeek R, Fonstein M, D'Souza M, Pusch GD, Maltsev N (1999) The use of gene clusters to infer functional coupling. Proc Natl Acad Sci USA 96:2896-2901

Penfield S, Li Y, Gilday AD, Graham S, Graham IA (2006) Arabidopsis ABA INSENSITIVE4 regulates lipid mobilization in the embryo and reveals repression of seed germination by the endosperm. Plant Cell 18:1887-1899

Penfield S, Rylott EL, Gilday AD, Graham S, Larson TR, Graham IA (2004) Reserve mobilization in the Arabidopsis endosperm fuels hypocotyl elongation in the dark, is independent of abscisic acid, and requires PHOSPHOENOLPYRUVATE CARBOXYKINASE1. Plant Cell 16:19

Pritchard SL, Charlton WL, Baker A, Graham IA (2002) Germination and storage reserve mobilization are regulated independently in Arabidopsis. Plant J 31:639-647

Raineri J, Ribichich KF, Chan RL (2015) The sunflower transcription factor HaWRKY76 confers drought and flood tolerance to Arabidopsis thaliana plants without yield penalty. Plant Cell Rep 34:2065-2080 
Rushton PJ, Macdonald H, Huttly AK, Lazarus CM, Hooley R (1995) Members of a new family of DNA-binding proteins bind to a conserved cis-element in the promoters of alpha-Amy2 genes. Plant Mol Biol 29:691-702

Rushton PJ, Somssich IE, Ringler P, Shen QJ (2010) WRKY transcription factors. Trends Plant Sci 15:247-258

Rylott EL, Gilday AD, Graham IA (2003) The gluconeogenic enzyme phosphoenolpyruvate carboxykinase in Arabidopsis is essential for seedling establishment. Plant Physiol 131:1834-1842

Rylott EL, Hooks MA, Graham IA (2001) Co-ordinate regulation of genes involved in storage lipid mobilization in Arabidopsis thaliana. Biochem Soc Trans 29:283-287

Schluttenhofer C, Yuan L (2015) Regulation of specialized metabolism by WRKY transcription factors. Plant Physiol 167:295-306

Siloto RM, Findlay K, Lopez-Villalobos A, Yeung EC, Nykiforuk CL, Moloney MM (2006) The accumulation of oleosins determines the size of seed oilbodies in Arabidopsis. Plant Cell 18:1961-1974

Smyth GK (2004) Linear models and empirical bayes methods for assessing differential expression in microarray experiments. Stat Appl Genet Mol Biol 3:1-25

Sun C, Palmqvist S, Olsson H, Borén M, Ahlandsberg S, Jansson C (2003) A novel WRKY transcription factor, SUSIBA2, participates in sugar signaling in barley by binding to the sugar-responsive elements of the iso 1 promoter. Plant Cell 15:2076-2092

Thelen JJ, Ohlrogge JB (2002) Metabolic engineering of fatty acid biosynthesis in plants. Metab Eng 4:12-21

Tripathi P, Rabara RC, Rushton PJ (2014) A systems biology perspective on the role of WRKY transcription factors in drought responses in plants. 
Usadel B, Poree F, Nagel A, Lohse M, Czedik-Eysenberg A, Stitt M (2009) A guide to using MapMan to visualize and compare Omics data in plants: a case study in the crop species, Maize. Plant Cell Environ 32:1211-1229

van Rooijen GJ, Moloney MM (1995) Plant seed oil-bodies as carriers for foreign proteins. Biotechnology (N Y) 13:72-77

Voinnet O, Rivas S, Mestre P, Baulcombe D (2003) An enhanced transient expression system in plants based on suppression of gene silencing by the p19 protein of tomato bushy stunt virus. Plant J 33:949-956

Xie Z, Zhang ZL, Hanzlik S, Cook E, Shen QJ (2007) Salicylic acid inhibits gibberellin-induced alpha-amylase expression and seed germination via a pathway involving an abscisic-acid-inducible $W R K Y$ gene. Plant Mol Biol 64:293-303

Xie Z, Zhang ZL, Zou X, Yang G, Komatsu S, Shen QJ (2006) Interactions of two abscisic-acid induced $W R K Y$ genes in repressing gibberellin signaling in aleurone cells. Plant J 46:231-242

Yan D, Duermeyer L, Leoveanu C, Nambara E (2014) The functions of the endosperm during seed germination. Plant Cell Physiol 55:1521-1533

Zentella R et al (2007) Global analysis of della direct targets in early gibberellin signaling in Arabidopsis. Plant Cell 19:3037-3057

Zhang Z-L, Xie Z, Zou X, Casaretto J, T-hD Ho, Shen QJ (2004) A rice $W R K Y$ gene encodes a transcriptional repressor of the gibberellin signaling pathway in aleurone cells. Plant Physiol 134:1500-1513 